Pacific

Journal of

Mathematics

\title{
HECKE-CLIFFORD ALGEBRAS \\ AND SPIN HECKE ALGEBRAS, II: \\ THE RATIONAL DOUBLE AFFINE TYPE
}

TA KHONGSAP AND WEIQIANG WANG 


\title{
HECKE-CLIFFORD ALGEBRAS AND SPIN HECKE ALGEBRAS, II: THE RATIONAL DOUBLE AFFINE TYPE
}

\author{
TA KHONGSAP AND WEIQIANG WANG
}

\begin{abstract}
This paper introduces the notion of rational spin double affine Hecke algebras (sDaHa) and rational double affine Hecke-Clifford algebras (DaHCa) associated to classical Weyl groups, and establishes the basic properties of these algebras, such as the PBW basis and Dunkl operator representations. We obtain an algebra isomorphism relating the rational DaHCa to the rational sDaHa. We further develop a link between the usual rational Cherednik algebra and the rational sDaHa by introducing a notion of rational covering double affine Hecke algebras.
\end{abstract}

$\begin{array}{ll}\text { 1. Introduction } & 73\end{array}$

2. The spin Weyl groups 76

3. Rational double affine Hecke-Clifford algebras (DaHCa) 79

4. The Dunkl operators for DaHCa 81

5. Rational spin double affine Hecke algebras (sDaHa) 87

6. The Dunkl operators for sDaHa 91

7. Rational covering double affine Hecke algebras (cDaHa) 93

8. Appendix: Proofs of several lemmas 96

$\begin{array}{ll}\text { References } & 102\end{array}$

\section{Introduction}

The rational Cherednik algebra (see Etingof and Ginzburg [2002] and also Drinfeld [1986] for a more general deformation construction) is a degenerate version of the double affine Hecke algebra [Cherednik 2005], and it admits a polynomial representation via the Dunkl operators [1989]. A similar degeneration in the case of affine Hecke algebras was introduced and studied earlier in [Drinfeld 1986] in the type $A$ case and by Lusztig in general [1989; 2002]. The rational Cherednik algebra has a rich representation theory and it affords various interesting connections to

MSC2000: $20 \mathrm{C} 08$.

Keywords: Cherednik algebras, Hecke algebras, symplectic reflection algebras, Clifford algebras, superalgebras, projective representation theory.

Wang is partially supported by an NSF grant. 
integrable systems, noncommutative geometry, and so on. We refer to Etingof [2007] and Rouquier [2005] for reviews and extensive references. The rational Cherednik algebra with one particular parameter being zero, denoted by $\ddot{\mathfrak{H}}_{W}$ in this introduction, is known to have a large center [Etingof and Ginzburg 2002]; see Gordon [2003].

In [Wang 2006], the second author introduced (degenerate) spin Hecke algebras of affine and double affine type as well as double affine Hecke-Clifford algebra, associated to I. Schur's spin symmetric group [1911]. These algebras were shown to be closely related to the affine Hecke-Clifford algebra of Nazarov [1997]. The spin affine Hecke algebras and affine Hecke-Clifford algebras associated to all classical Weyl groups have been recently constructed by the authors [Khongsap and Wang 2008].

In this paper we shall construct three classes of closely related (super) algebras associated to each classical finite Weyl group $W$ : the rational double affine Hecke-Clifford algebra (DaHCa) $\ddot{\mathfrak{H}}_{W}^{\mathfrak{c}}$, the rational spin double affine Hecke algebra (sDaHa) $\ddot{\mathfrak{H}}_{W}^{-}$, and the rational covering double affine Hecke algebra (cDaHa) $\ddot{\mathfrak{H}}_{W}^{\sim}$. We show that the algebras $\ddot{\mathfrak{H}}_{W}^{\mathfrak{c}}$ and $\ddot{\mathfrak{H}}_{W}^{-}$are Morita superequivalent (in the terminology of [Wang 2007]) and that $\ddot{\mathfrak{H}}_{W}^{\sim}$ has both the rational Cherednik algebra $\ddot{\mathfrak{H}}_{W}$ and the sDaHa $\ddot{\mathfrak{H}}_{W}^{-}$as its natural quotients. We further establish some basic properties including the PBW basis theorem and Dunkl operator realizations of these algebras.

We expect that these algebras afford very interesting representation theory and connections with noncommutative geometry.

Morris [1976] and Khongsap and Wang [2008] considered a double cover $\widetilde{W}$ of the finite Weyl group $W$ associated to a distinguished 2-cocycle

$$
1 \rightarrow \mathbb{Z}_{2} \longrightarrow \widetilde{W} \longrightarrow W \rightarrow 1 \text {. }
$$

Denote $\mathbb{Z}_{2}=\{1, z\}$. From now on, let $W$ be one of the classical Weyl groups. In this paper, we define the algebras $\ddot{\mathfrak{H}}_{W}^{\mathfrak{c}}, \ddot{\mathfrak{H}}_{W}^{-}$and $\ddot{\mathfrak{H}}_{W}^{\sim}$ for every $W$ of type $A_{n-1}, D_{n}, B_{n}$ in terms of explicit generators and relations, where the number of parameters in each of these algebras is the number of conjugacy classes of reflections in $W$. The compatibility among the defining relations for $\ddot{\mathfrak{H}}_{W}^{\mathfrak{c}}$ (which would imply the PBW basis property and similar compatibility for $\ddot{\mathfrak{H}}_{W}^{-}$and $\ddot{\mathfrak{H}}_{W}^{\sim}$ when combined with other results) requires lengthy but elementary case-by-case verifications. In a suitable sense, the defining relations are naturally and uniquely dictated by the compatibility of these relations.

As is well known, the rational Cherednik algebra $\ddot{\mathfrak{H}}_{W}$ has a triangular decomposition with the group algebra $\mathbb{C} W$ as its middle term. We show that the algebras $\ddot{\mathfrak{H}}_{W}^{\mathrm{c}}$, $\ddot{\mathfrak{H}}_{W}^{-}$and $\ddot{\mathfrak{H}}_{W}^{\sim}$ also afford triangular decompositions which contain $\mathscr{C}_{n} \rtimes \mathbb{C} W, \mathbb{C} W^{-}$ and $\mathbb{C} \widetilde{W}$ respectively as the middle terms, where $\mathscr{C}_{n}$ denotes the Clifford algebra of the reflection representation of $W$ with a natural $W$-action. For instance, the 
rational DaHCa $\ddot{\mathfrak{H}}_{W}^{\mathfrak{c}}$ and sDaHa $\ddot{\mathfrak{H}}_{W}^{-}$have the triangular decompositions

$$
\begin{aligned}
& \ddot{\mathfrak{H}}_{W}^{\mathfrak{c}} \cong \mathbb{C}\left[x_{1}, \ldots, x_{n}\right] \otimes\left(\mathscr{C}_{n} \rtimes \mathbb{C} W\right) \otimes \mathbb{C}\left[y_{1}, \ldots, y_{n}\right], \\
& \ddot{\mathfrak{H}}_{W}^{-} \cong \mathscr{C}\left[\xi_{1}, \ldots, \xi_{n}\right] \otimes \mathbb{C} W^{-} \otimes \mathbb{C}\left[y_{1}, \ldots, y_{n}\right],
\end{aligned}
$$

where $\mathscr{C}\left[\xi_{1}, \ldots, \xi_{n}\right]$ is a noncommutative algebra with $\xi_{i} \xi_{j}=-\xi_{j} \xi_{i}$ for $i \neq j$. The relations between $\mathbb{C} W^{-}$and $\mathscr{C}\left[\xi_{1}, \ldots, \xi_{n}\right]$ involve subtle signs similar to those appearing in the spin affine Hecke algebras defined in [Khongsap and Wang 2008; Wang 2006].

We further show that the algebras $\ddot{\mathfrak{H}}_{W}^{c}$ and $\ddot{\mathfrak{H}}_{W}^{-}$have large centers which contain $\mathbb{C}\left[y_{1}, \ldots, y_{n}\right]^{W}$ and $\mathbb{C}\left[x_{1}^{2}, \ldots, x_{n}^{2}\right]^{W}$ (and $\mathbb{C}\left[\xi_{1}^{2}, \ldots, \xi_{n}^{2}\right]^{W}$ respectively) as subalgebras. In particular, the algebras $\ddot{\mathfrak{H}}_{W}^{\mathfrak{c}}$ and $\ddot{\mathfrak{H}}_{W}^{-}$are module-finite over their centers.

The group algebra $\mathbb{C} W$ and the spin Weyl group algebra $\mathbb{C} W^{-}$appear as natural quotients of $\mathbb{C} \widetilde{W}$ by the ideals $\langle z \mp 1\rangle$ respectively. We show that these quotient maps, denoted by $\Upsilon_{ \pm}$, extend to the setup of double affine Hecke algebras. All these statements can be summarized in the following commutative diagram, with the vertical arrows being natural inclusions:

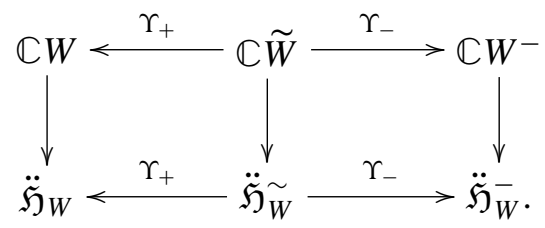

In [Khongsap and Wang 2008], we established a superalgebra isomorphism

$$
\Phi: \mathscr{C}_{n} \rtimes \mathbb{C} W \stackrel{\simeq}{\longrightarrow} \mathscr{C}_{n} \otimes \mathbb{C} W^{-}
$$

(which actually holds also for exceptional Weyl groups), generalizing the type $A$ result of Sergeev and Yamaguchi. In this paper, we shall establish a Morita superequivalence between $\ddot{\mathfrak{H}}_{W}^{\mathfrak{c}}$ and $\ddot{\mathfrak{H}}_{W}^{-}$, or more explicitly, a superalgebra isomorphism between $\ddot{\mathfrak{H}}_{W}^{\mathfrak{c}}$ and the tensor algebra $\mathscr{C}_{n} \otimes \ddot{\mathfrak{H}}_{W}^{-}$that extends the isomorphism $\Phi$ (see [Wang 2006] for the type $A$ case). This can be summarized conveniently in the following commutative diagram, with the vertical arrows being natural inclusions:

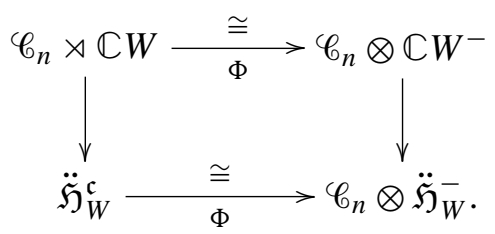

As our constructions in a way rely on a choice of orthonormal basis of $\mathfrak{h}$, they do not seem to be easily extendable to the exceptional Weyl groups. Also, in contrast to the setup of rational Cherednik algebras in [Etingof and Ginzburg 2002], 
our Hecke algebras do not seem to afford an extra parameter in a natural way to trivialize their center.

There has been another attempt (see [Chmutova 2005]) to generalize the rational Cherednik algebras and more generally symplectic reflection algebras by adding a twist with a 2-cocycle of a finite group. But the approach there does not produce intrinsically interesting new algebras with nontrivial 2-cocycles of the Weyl groups as does our approach.

The paper is organized as follows. In Section 2, we recall some facts about the distinguished double covers of the Weyl groups. For more detailed treatment, consult [Khongsap and Wang 2008]. We introduce in Section 3 the rational DaHCa $\ddot{\mathfrak{H}}_{W}^{\mathfrak{c}}$ and establish its PBW basis property. Section 4 obtains the Dunkl operator representations of $\ddot{\mathfrak{H}}_{W}^{\mathfrak{c}}$. Sections 5 and 6 are the counterparts for the sDaHa $\ddot{\mathfrak{H}}_{W}^{-}$of Section 3 and 4, respectively. In addition, Section 5 establishes the superalgebra isomorphism $\Phi$ relating the sDaHa and DaHCa. Finally, Section 7 introduces the rational cDaHa $\ddot{\mathfrak{H}}_{W}^{\sim}$, which provides a link between the sDaHa $\ddot{\mathfrak{H}}_{W}^{-}$and the usual DaHa $\ddot{\mathfrak{H}}_{W}$. Finally, in the appendix (Section 8), we present the proofs of several lemmas in Section 3 and 4.

\section{The spin Weyl groups}

In this section, we recall from [Khongsap and Wang 2008] some preliminary setups which lead to Theorem 2.1 below, but here we will restrict ourselves to classical Weyl groups only, as this is all we need subsequently.

2.1. A double covering of Weyl groups. Let $W$ be an (irreducible) finite Weyl group of classical type (that is, of type $A, B, D$ ) with the presentation

$$
\left.\left\langle s_{1}, \ldots, s_{n}\right|\left(s_{i} s_{j}\right)^{m_{i j}}=1, m_{i i}=1, m_{i j}=m_{j i} \in \mathbb{Z}_{\geq 2} \text { for } i \neq j\right\rangle .
$$

The integers $m_{i j}$ are specified by the Coxeter-Dynkin diagrams whose vertices correspond to the generators of $W$ below. By convention, we only mark the edge connecting $i$ and $j$ with $m_{i j} \geq 4$. We have $m_{i j}=3$ for $i \neq j$ connected by an unmarked edge, and $m_{i j}=2$ if $i$ and $j$ are not connected by an edge.

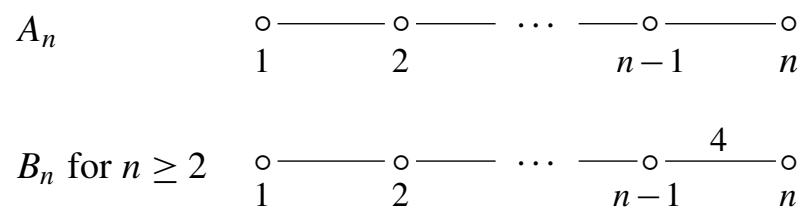




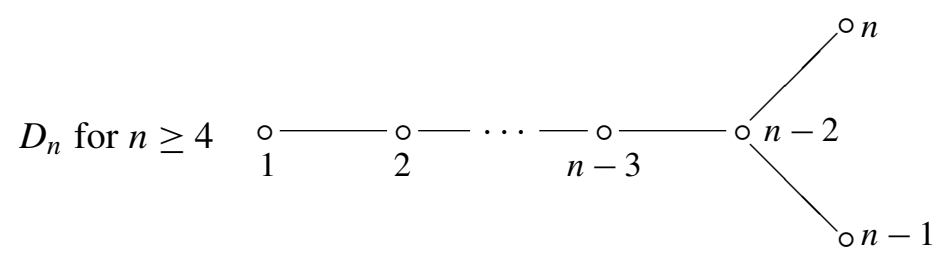

We shall be concerned about a distinguished double covering $\widetilde{W}$ of $W$ :

$$
1 \rightarrow \mathbb{Z}_{2} \longrightarrow \widetilde{W} \longrightarrow W \rightarrow 1
$$

We let $\mathbb{Z}_{2}=\{1, z\}$ and denote by $\tilde{t}_{i}$ a fixed preimage of the generators $s_{i}$ of $W$ for each $i$. The group $\widetilde{W}$ is generated by $z, \tilde{t}_{1}, \ldots, \tilde{t}_{n}$ with relations (besides the obvious relation that $z$ is central of order 2) listed in Table 1, which corresponds to setting the $\alpha_{i}$ for all $i$ in Karpilovsky [1987, Table 7.1] to be $z$.

The quotient algebra $\mathbb{C} W^{-}:=\mathbb{C} \widetilde{W} /\langle z+1\rangle$ of $\mathbb{C} \widetilde{W}$ by the ideal generated by $z+1$ will be called the spin Weyl group algebra associated to $W$. Denote by $t_{i} \in \mathbb{C} W^{-}$ the image of $\tilde{t}_{i}$. The spin Weyl group algebra $\mathbb{C} W^{-}$has a natural superalgebra (that is, $\mathbb{Z}_{2}$-graded algebra) structure by letting each $t_{i}$ be odd. The algebra $\mathbb{C} W^{-}$ is generated by $t_{1}, \ldots, t_{n}$ with the labeling as in the Coxeter-Dynkin diagrams and the explicit relations summarized in Table 2.

By definition, the quotient by the ideal $\langle z-1\rangle$ of the group algebra $\mathbb{C} \widetilde{W}$ is isomorphic to $\mathbb{C} W$.

2.2. A superalgebra isomorphism. Denote by $\mathfrak{h}=\mathbb{C}^{n}$ the natural representation (respectively the reflection representation) of the Weyl group $W$ of type $A_{n-1}$ (respectively of type $B_{n}$ and $D_{n}$ ). Note that $\mathfrak{h}$ carries a $W$-invariant nondegenerate

\begin{tabular}{cl}
\hline$W$ & Defining Relations for $\widetilde{W}$ \\
\hline \multirow{3}{*}{$A_{n}$} & $\tilde{t}_{i}^{2}=1$ for $1 \leq i \leq n$, \\
& $\tilde{t}_{i} \tilde{t}_{i+1} \tilde{t}_{i}=\tilde{t}_{i+1} \tilde{t}_{i} \tilde{t}_{i+1}$ for $1 \leq i \leq n-1$, \\
& $\tilde{t}_{i} \tilde{t}_{j}=z \tilde{t}_{j} \tilde{t}_{i}$ if $m_{i j}=2$. \\
\hline \multirow{3}{*}{$B_{n}$} & $\tilde{t}_{i}^{2}=1$ for $1 \leq i \leq n, \quad \tilde{t}_{i} \tilde{t}_{i+1} \tilde{t}_{i}=\tilde{t}_{i+1} \tilde{t}_{i} \tilde{t}_{i+1}$ for $1 \leq i \leq n-2$, \\
$(n \geq 2)$ & $\tilde{t}_{i} \tilde{t}_{j}=z \tilde{t}_{j} \tilde{t}_{i}$ for $1 \leq i<j \leq n-1, m_{i j}=2$, \\
& $\tilde{t}_{i} \tilde{t}_{n}=z \tilde{t}_{n} \tilde{t}_{i}$ for $1 \leq i \leq n-2$, \\
& $\left(\tilde{t}_{n-1} \tilde{t}_{n}\right)^{2}=z\left(\tilde{t}_{n} \tilde{t}_{n-1}\right)^{2}$. \\
& $\tilde{t}_{i}^{2}=1$ for $1 \leq i \leq n$, \\
$(n \geq 4)$ & $\tilde{t}_{i} \tilde{t}_{j} \tilde{t}_{i}=\tilde{t}_{j} \tilde{t}_{i} \tilde{t}_{j}$ if $m_{i j}=3$, \\
\hline & $\tilde{t}_{i} \tilde{t}_{j}=z \tilde{t}_{j} \tilde{t}_{i}$ for $1 \leq i<j \leq n, m_{i j}=2$. \\
\hline
\end{tabular}

Table 1. The defining relations of $\widetilde{W}$. 


\begin{tabular}{cl}
\hline Type of $W$ & Defining Relations for $\mathbb{C} W^{-}$ \\
\hline$A_{n}$ & $t_{i}^{2}=1, t_{i} t_{i+1} t_{i}=t_{i+1} t_{i} t_{i+1}$, \\
& $\left(t_{i} t_{j}\right)^{2}=-1$ if $|i-j|>1$. \\
\hline & $t_{1}, \ldots, t_{n-1}$ satisfy these relations for $\mathbb{C} W_{A_{n-1}}^{-}:$ \\
$B_{n}$ & $t_{n}^{2}=1,\left(t_{i} t_{n}\right)^{2}=-1$ if $i \neq n-1, n$, \\
& $\left(t_{n-1} t_{n}\right)^{4}=-1$. \\
\hline \multirow{3}{*}{$D_{n}$} & $t_{1}, \ldots, t_{n-1}$ satisfy these relations for $\mathbb{C} W_{A_{n-1}}^{-}:$ \\
& $t_{n}^{2}=1,\left(t_{i} t_{n}\right)^{2}=-1$ if $i \neq n-2, n$, \\
& $t_{n-2} t_{n} t_{n-2}=t_{n} t_{n-2} t_{n}$ \\
\hline
\end{tabular}

Table 2. The defining relations of $\mathbb{C} W^{-}$.

bilinear form $(\cdot, \cdot)$, which gives rise to an identification $\mathfrak{h}^{*} \cong \mathfrak{h}$ and also a bilinear form on $\mathfrak{h}^{*}$ which will be again denoted by $(\cdot, \cdot)$.

Denote by $\mathscr{C}_{n}$ the Clifford algebra associated to $(\mathfrak{h},(\cdot, \cdot))$. We shall denote by $\left\{c_{i}\right\}$ the generators in $\mathscr{C}_{n}$ corresponding to a standard orthonormal basis $\left\{e_{i}\right\}$ of $\mathbb{C}^{n}$, and denote by $\left\{\beta_{i}\right\}$ the elements of $\mathscr{C}_{n}$ corresponding to the simple roots $\left\{\alpha_{i}\right\}$, normalized with $\beta_{i}^{2}=1$. More explicitly, $\mathscr{C}_{n}$ is generated by $c_{1}, \ldots, c_{n}$ subject to the relations

$$
c_{i}^{2}=1 \quad \text { and } \quad c_{i} c_{j}=-c_{j} c_{i} \quad \text { for } i \neq j .
$$

For type $A_{n-1}$, we have $\beta_{i}=\left(c_{i}-c_{i+1}\right) / \sqrt{2}$ for $1 \leq i \leq n-1$. For type $B_{n}$, we have an additional $\beta_{n}=c_{n}$, and $\beta_{n}=\left(c_{n-1}+c_{n}\right) / \sqrt{2}$ for type $D_{n}$.

The action of $W$ on $\mathfrak{h}$ and $\mathfrak{h}^{*}$ preserves the bilinear form $(\cdot, \cdot)$ and thus it acts by automorphisms of the algebra $\mathscr{C}_{n}$. This gives rise to a semidirect product $\mathscr{C}_{n} \rtimes \mathbb{C} W$. Moreover, the algebra $\mathscr{C}_{n} \rtimes \mathbb{C} W$ naturally inherits the superalgebra structure by letting elements in $W$ be even and each $c_{i}$ be odd.

Given two superalgebras $\mathscr{A}$ and $\mathscr{B}$, we view the tensor product of superalgebras $\mathscr{A} \otimes \mathscr{B}$ as a superalgebra with multiplication defined by

$$
(a \otimes b)\left(a^{\prime} \otimes b^{\prime}\right)=(-1)^{|b|\left|a^{\prime}\right|}\left(a a^{\prime} \otimes b b^{\prime}\right) \quad \text { for } a, a^{\prime} \in \mathscr{A} \text { and } b, b^{\prime} \in \mathscr{B},
$$

where $|b|$ denotes the $\mathbb{Z}_{2}$-degree of $b$, and so on. Also, we shall use shorthand notation $a b$ for $(a \otimes b) \in \mathscr{A} \otimes \mathscr{B}$ with $a=a \otimes 1$ and $b=1 \otimes b$.

Theorem 2.1 [Khongsap and Wang 2008]. We have an isomorphism

$$
\Phi: \mathscr{C}_{n} \rtimes \mathbb{C} W \stackrel{\simeq}{\longrightarrow} \mathscr{C}_{n} \otimes \mathbb{C} W^{-}
$$

of superalgebras extending the identity map on $\mathscr{C}_{n}$ and sends $s_{i} \mapsto-\sqrt{-1} \beta_{i} t_{i}$. The inverse map $\Psi$ is the extension of the identity map on $\mathscr{C}_{n}$ that sends $t_{i} \mapsto \sqrt{-1} \beta_{i} s_{i}$. 
(In the terminology of [Wang 2007], the superalgebras $\mathscr{C}_{n} \rtimes \mathbb{C} W$ and $\mathbb{C} W^{-}$are Morita superequivalent.)

Remark 2.2. Theorem 2.1 was formulated and proved in [Khongsap and Wang 2008] for every finite Weyl group including the exceptional types, and the type $A$ case was treated by Sergeev and Yamaguchi.

\section{Rational double affine Hecke-Clifford algebras (DaHCa)}

In this section, we introduce the rational double affine Hecke-Clifford algebras associated to the Weyl group $W$ of type $A, D$ and $B$, and then establish the PBW property. The type $A$ case was treated in [Wang 2006].

\section{The definition of the algebras $\ddot{\mathfrak{H}}_{W}^{\mathfrak{c}}$.}

The algebra $\ddot{\mathfrak{H}}_{W}^{\mathfrak{c}}$ of type $A_{n-1}$. We will make the identifications

$$
\mathbb{C}\left[\mathfrak{h}^{*}\right] \cong \mathbb{C}\left[x_{1}, \ldots, x_{n}\right] \text { and } \mathbb{C}[\mathfrak{h}] \cong \mathbb{C}\left[y_{1}, \ldots, y_{n}\right],
$$

where the $x_{i}$ and $y_{i}$ correspond to the standard orthonormal basis $\left\{e_{i}\right\}$ for $\mathfrak{h}^{*}$ and its dual basis $\left\{e_{i}^{*}\right\}$ for $\mathfrak{h}$.

The following algebra $\ddot{\mathfrak{H}}_{A_{n-1}}^{\mathfrak{c}}$ was introduced in [Wang 2006] under the notation $\mathscr{A}_{u}$. We recall it for convenience and usage in the subsequent subsections. For $x, y$ in an algebra $A$, we denote as usual that $[x, y]=x y-y x \in A$.

Definition 3.1. Let $u \in \mathbb{C}$ and $W=W_{A_{n-1}} \equiv S_{n}$. The rational double affine HeckeClifford algebra (DaHCa) of type $A_{n-1}$, denoted by $\ddot{\mathfrak{H}}_{W}^{\mathfrak{c}}$ or $\ddot{\mathfrak{H}}_{A_{n-1}}^{\mathfrak{c}}$, is the algebra generated by $x_{i}, y_{i}$ and $c_{i}$ for $1 \leq i \leq n$ and $W$, subject to the relation (2-2) among the $c_{i}$ and the following relations (where we identify $\mathfrak{h}^{*}=\mathbb{C} x_{1}+\cdots+\mathbb{C} x_{n}$ and $\left.\mathfrak{h}=\mathbb{C} y_{1}+\cdots+\mathbb{C} y_{n}\right):$

$$
\begin{cases}x_{i} x_{j}=x_{j} x_{i}, y_{i} y_{j}=y_{j} y_{i}, y_{i} c_{j}=c_{j} y_{i} & \text { for all } i, j, \\ x_{i} c_{i}=-c_{i} x_{i}, x_{i} c_{j}=c_{j} x_{i} & \text { for } i \neq j, \\ w x w^{-1}=w(x) & \text { for all } x \in \mathfrak{h}^{*} \text { and } w \in W, \\ w y w^{-1}=w(y) & \text { for all } y \in \mathfrak{h} \text { and } w \in W, \\ w c w^{-1}=w(c) & \text { for all } c \in \mathscr{C}_{n} \text { and } w \in W,\end{cases}
$$

and

$$
\begin{aligned}
{\left[y_{j}, x_{i}\right] } & =u\left(1+c_{j} c_{i}\right) s_{j i} \quad \text { for } i \neq j, \\
{\left[y_{i}, x_{i}\right] } & =-u \sum_{k \neq i}\left(1+c_{k} c_{i}\right) s_{k i} .
\end{aligned}
$$

Alternatively, we may view $u$ as a formal variable and $\ddot{\mathfrak{H}}_{W}^{\mathfrak{c}}$ as a $\mathbb{C}(u)$-algebra. Similar remarks apply to all $\mathrm{DaHCa}$, sDaHa, and $\mathrm{cDaHa}$ introduced in this paper. 
The algebra $\ddot{\mathfrak{H}}_{W}^{\mathfrak{c}}$ of type $D_{n}$. Let $W=W_{D_{n}}$. Regarding elements in $W$ as even signed permutations of $1,2, \ldots, n$ as usual, we identify the generators $s_{i} \in W$ for $1 \leq i \leq n-1$ with transposition $(i, i+1)$, and $s_{n} \in W$ with the transposition of $(n-1, n)$ coupled with the sign changes at $n-1$ and $n$. For $1 \leq i \neq j \leq n$, we denote by $s_{i j} \equiv(i, j) \in W$ the transposition of $i$ and $j$, and by $\bar{s}_{i j} \equiv \overline{(i, j)} \in W$ the transposition of $i$ and $j$ coupled with the sign changes at $i, j$. By convention,

$$
\bar{s}_{n-1, n} \equiv \overline{(n-1, n)}=s_{n} \quad \text { and } \quad \bar{s}_{i j} \equiv \overline{(i, j)}=s_{j n} s_{i, n-1} s_{n} s_{i, n-1} s_{j n} .
$$

Definition 3.2. Let $u \in \mathbb{C}$ and $W=W_{D_{n}}$. The rational double affine Hecke-Clifford algebra of type $D_{n}$, denoted by $\ddot{\mathfrak{H}}_{W}^{\mathfrak{c}}$ or $\ddot{\mathfrak{H}}_{D_{n}}^{\mathfrak{c}}$, is the algebra generated by $x_{i}, y_{i}$ and $c_{i}$ for $1 \leq i \leq n$ and $W$, subject to the relation (2-2) among the $c_{i}$, (3-1) with the current $W$, and the relations

$$
\begin{aligned}
{\left[y_{j}, x_{i}\right] } & =u\left(\left(1+c_{j} c_{i}\right) s_{i j}-\left(1-c_{j} c_{i}\right) \bar{s}_{i j}\right) \quad \text { for } i \neq j, \\
{\left[y_{i}, x_{i}\right] } & =-u \sum_{k \neq i}\left(\left(1+c_{k} c_{i}\right) s_{k i}+\left(1-c_{k} c_{i}\right) \bar{s}_{k i}\right) .
\end{aligned}
$$

The algebra $\ddot{\mathfrak{H}}_{W}^{\mathfrak{c}}$ of type $B_{n}$. Let $W=W_{B_{n}}$. We identify $W$ as usual with the signed permutations on $1, \ldots, n$. Regarding $W_{D_{n}}$ as a subgroup of $W$, we have $s_{i j}, \bar{s}_{i j} \in W$ for $1 \leq i \neq j \leq n$. Further denote by $\tau_{i} \equiv \overline{(i)} \in W$ the sign change at $i$ for $1 \leq i \leq n$. By definition, we have $\tau_{n} \equiv \overline{(n)}=s_{n}$ and $\tau_{i} \equiv \overline{(i)}=s_{i n} s_{n} s_{i n}$.

Definition 3.3. Let $u, v \in \mathbb{C}$, and $W=W_{B_{n}}$. The rational double affine HeckeClifford algebra of type $B_{n}$, denoted by $\ddot{\mathfrak{H}}_{W}^{\mathfrak{c}}$ or $\ddot{\mathfrak{H}}_{B_{n}}^{\mathfrak{c}}$, is the algebra generated by $x_{i}$, $y_{i}$ and $c_{i}$ for $1 \leq i \leq n$ and $W$, subject to the relations (2-2) for the $c_{i}$, (3-1) with the current $W$, and the relations

$$
\begin{aligned}
{\left[y_{j}, x_{i}\right] } & =u\left(\left(1+c_{j} c_{i}\right) s_{i j}-\left(1-c_{j} c_{i}\right) \bar{s}_{i j}\right) \quad \text { for } i \neq j, \\
{\left[y_{i}, x_{i}\right] } & =-u \sum_{k \neq i}\left(\left(1+c_{k} c_{i}\right) s_{k i}+\left(1-c_{k} c_{i}\right) \bar{s}_{k i}\right)-\sqrt{2} v \tau_{i} .
\end{aligned}
$$

When it is necessary to indicate the dependence of the algebra $\ddot{\mathfrak{H}}_{W}^{\mathfrak{c}}$ on $u$ and $v$, we will write $\ddot{\mathfrak{H}}_{W}^{\mathfrak{c}}(u, v)$ for $\ddot{\mathfrak{H}}_{W}^{\mathfrak{c}}$.

Remark 3.4. The factor $\sqrt{2}$ in (3-4b) is inserted to make the definition of $\ddot{\mathfrak{H}}_{B_{n}}^{\mathfrak{c}}$ compatible with the notion of sDaHa $\ddot{\mathfrak{H}}_{B_{n}}^{-}$below under a Morita superequivalence $\Phi$. See Theorem 5.5.

The PBW basis for $\ddot{\mathfrak{H}}_{W}^{\mathfrak{c}}$. For any classical Weyl group $W$, the algebra $\ddot{\mathfrak{H}}_{W}^{\mathfrak{c}}$ is a superalgebra by letting elements of $W$ and $x_{i}$ and $y_{i}$ for all $i$ be even, and letting each $c_{i}$ be odd.

Theorem 3.5. Let $W$ be $W_{A_{n-1}}, W_{D_{n}}$ or $W_{B_{n}}$. The multiplication of the subalgebras $\mathbb{C}\left[\mathfrak{h}^{*}\right], \mathbb{C}[\mathfrak{h}], \mathscr{C}_{n}$, and $\mathbb{C} W$ induces a vector space isomorphism

$$
\mathbb{C}\left[\mathfrak{h}^{*}\right] \otimes \mathscr{C}_{n} \otimes \mathbb{C} W \otimes \mathbb{C}[\mathfrak{h}] \stackrel{\simeq}{\longrightarrow} \ddot{\mathfrak{H}}_{W}^{\mathfrak{c}} .
$$


Equivalently, the elements $\left\{x^{\alpha} c^{\epsilon} w y^{\gamma} \mid \alpha, \gamma \in \mathbb{Z}_{+}^{n}, \in \in \mathbb{Z}_{2}^{n}, w \in W\right\}$ form a linear basis for $\ddot{\mathfrak{H}}_{W}^{\mathfrak{c}}$ (the PBW basis).

Proof. Recall that $W$ acts diagonally on $V=\mathfrak{h}^{*} \oplus \mathfrak{h}$. The proof is similar to the proof of [Etingof and Ginzburg 2002, Theorem 1.3] with one crucial modification, as first observed in [Wang 2006].

Clearly $K:=\mathscr{C}_{n} \rtimes \mathbb{C} W$ is a semisimple algebra. Observe that $E:=V \otimes_{\mathbb{C}} K$ is a natural $K$-bimodule (even though $V$ is not) with the right $K$-module structure on $E$ given by right multiplication and the left $K$-module structure on $E$ by letting

$$
\begin{aligned}
w \cdot(v \otimes a) & =v^{w} \otimes w a, \\
c_{i} .\left(x_{j} \otimes a\right) & =(-1)^{\delta_{i j}} x_{j} \otimes\left(c_{i} a\right), \\
c_{i} \cdot\left(y_{j} \otimes a\right) & =y_{j} \otimes\left(c_{i} a\right),
\end{aligned}
$$

where $v \in V, w \in W$ and $a \in K$.

The rest of the proof can proceed in the same way as in the proof of [Etingof and Ginzburg 2002, Theorem 1.3]. It boils down to the verifications in Lemmas 3.7, 3.8 and 3.9 below of the conjugation invariance (by $c_{i}$ and $W$ ) of the defining relations (3-2), (3-3), or (3-4) for type $A, D$, or $B$, respectively, and of the Jacobi identities among the generators $x_{i}$ and $y_{i}$.

Remark 3.6. Note that $\mathscr{C}_{n} \rtimes \mathbb{C W}$ is actually a subalgebra of $\ddot{\mathfrak{H}}_{W}^{\mathfrak{c}}$ and the tensor product in the above theorem indicates that $\ddot{\mathfrak{H}}_{W}^{\mathfrak{c}}$ has the structure of an algebra with triangular decomposition:

$$
\ddot{\mathfrak{H}}_{W}^{\mathfrak{c}} \cong \mathbb{C}\left[\mathfrak{h}^{*}\right] \otimes\left(\mathfrak{C}_{n} \rtimes \mathbb{C} W\right) \otimes \mathbb{C}[\mathfrak{h}] .
$$

We will prove Lemmas 3.7, 3.8 and 3.9 in the appendix.

Lemma 3.7. Let $W=W_{A_{n-1}}, W_{D_{n}}$, or $W_{B_{n}}$. Then the relations (3-2), (3-3), or (3-4) are invariant under the conjugation by $c_{i}$ for $1 \leq i \leq n$.

Lemma 3.8. The relations (3-2), (3-3), or (3-4) are invariant under the conjugation by elements in $W_{A_{n-1}}, W_{D_{n}}$, or $W_{B_{n}}$, respectively.

Lemma 3.9. Let $W=W_{A_{n-1}}, W_{D_{n}}$, or $W_{B_{n}}$. Then the Jacobi identity holds for any triple among $x_{i}$ and $y_{i}$ in $\ddot{\mathfrak{H}}_{W}^{\mathfrak{c}}$ for $1 \leq i \leq n$.

\section{The Dunkl operators for DaHCa}

The Dunkl representations. The algebra $\ddot{\mathfrak{H}}_{W}^{\mathfrak{c}}$ is a superalgebra by letting elements of $W$ and $x_{i}$ and $y_{i}$ for all $i$ be even, and letting each $c_{i}$ be odd. Recall that $\ddot{\mathfrak{H}}_{W}^{\mathfrak{c}}$ admits the triangular decomposition $\ddot{\mathfrak{H}}_{W}^{\mathfrak{c}} \cong \mathbb{C}\left[\mathfrak{h}^{*}\right] \otimes K \otimes \mathbb{C}[\mathfrak{h}]$, where we have written $K=\mathscr{C}_{n} \rtimes \mathbb{C} W$. In contrast to the usual DaHa $\ddot{\mathfrak{H}}_{W}$, the DaHCa $\ddot{\mathfrak{H}}_{W}^{\mathfrak{c}}$ has no automorphism that switches the subalgebras $\mathbb{C}[\mathfrak{h}]$ and $\mathbb{C}\left[\mathfrak{h}^{*}\right]$. Denote by $\mathfrak{H}_{x}$ and $\mathfrak{H}_{y}$ 
the subalgebras of $\ddot{\mathfrak{H}}_{W}^{\mathfrak{c}}$ generated by $K$ and $x_{1}, \ldots, x_{n}$, and generated by $K$ and $y_{1}, \ldots, y_{n}$, respectively.

A $K$-module $M$ can be extended to either an $\mathfrak{H}_{x}$-module or an $\mathfrak{H}_{y}$-module by demanding the action of the $x_{i}$ and the $y_{i}$ to be trivial, respectively. We define the induced $\ddot{\mathfrak{H}}_{W}^{\mathfrak{c}}$-modules

$$
M_{x}:=\ddot{\mathfrak{H}}_{W}^{\mathfrak{c}} \otimes_{\mathfrak{H}_{x}} M \quad \text { and } \quad M_{y}:=\ddot{\mathfrak{H}}_{W}^{\mathfrak{c}} \otimes_{\mathfrak{H}_{y}} M .
$$

Below we will always use the vector space identifications

$$
M_{x}=\mathbb{C}\left[y_{1}, \ldots, y_{n}\right] \otimes M \quad \text { and } \quad M_{y}=\mathbb{C}\left[x_{1}, \ldots, x_{n}\right] \otimes M .
$$

The action of $\ddot{\mathfrak{H}}_{W}^{\mathfrak{c}}$ on $M_{x}$ (respectively $M_{y}$ ) is transferred to $\mathbb{C}\left[y_{1}, \ldots, y_{n}\right] \otimes M$ (respectively $\mathbb{C}\left[x_{1}, \ldots, x_{n}\right] \otimes M$ ) as follows. On $\mathbb{C}\left[y_{1}, \ldots, y_{n}\right] \otimes M, K$ acts diagonally. More explicitly, $\mathrm{K}$ acts on $\mathbb{C}\left[x_{1}, \ldots, x_{n}\right] \otimes M$ by

$$
w \cdot\left(x_{j} \otimes m\right)=x_{j}^{w} \otimes w m \quad \text { and } \quad c_{i} \cdot\left(x_{j} \otimes m\right)=(-1)^{\delta_{i j}} x_{j} \otimes c_{i} m,
$$

where $c_{i} \in \mathscr{C}_{n}$ and $w \in W$. Moreover, $y_{i}$ acts by left multiplication in the first tensor factor, and the action of $x_{i}$ is given by the so-called Dunkl operators (which are generalizations of those in [Dunkl 1989]). Similarly, on $\mathbb{C}\left[x_{1}, \ldots, x_{n}\right] \otimes M$, $x_{i}$ acts by left multiplication, and $y_{i}$ acts by another version of Dunkl operators. In the remainder of this section we shall describe these Dunkl operators explicitly.

Remark 4.1. A canonical choice for a $K$-module is $\mathscr{C}_{n}$, whose $K$-module structure is defined by letting $\mathscr{C}_{n}$ act by left multiplication and letting $W$ act as usual; see Section 2.2.

The Dunkl operators for $\ddot{\mathfrak{H}}_{A_{n-1}}^{\mathfrak{c}}$. We first prepare a few lemmas. We shall denote the action of $\sigma \in W$ on $\mathbb{C}[\mathfrak{h}]$ and $\mathbb{C}\left[\mathfrak{h}^{*}\right]$ by $f \mapsto f^{\sigma}$.

Lemma 4.2. Let $W=W_{A_{n-1}}$. Then the following hold in $\ddot{\mathfrak{H}}_{W}^{\mathfrak{c}}$ for $l \in \mathbb{Z}_{+}$and $i \neq j$ :

$$
\begin{aligned}
& {\left[y_{i}, x_{j}^{l}\right]=u\left(\frac{x_{j}^{l}-x_{i}^{l}}{x_{j}-x_{i}}+\frac{x_{j}^{l}-\left(-x_{i}\right)^{l}}{x_{j}+x_{i}} c_{i} c_{j}\right) s_{i j},} \\
& {\left[y_{i}, x_{i}^{l}\right]=-u \sum_{k \neq i}\left(\frac{x_{i}^{l}-x_{k}^{l}}{x_{i}-x_{k}}+\frac{x_{i}^{l}-\left(-x_{k}\right)^{l}}{x_{i}+x_{k}} c_{k} c_{i}\right) s_{k i} .}
\end{aligned}
$$

It is understood here and in similar ratios of operators below that $h / g=(1 / g) \cdot h$.

Proof. This lemma is a type $A$ counterpart of Lemma 4.8 for type $B$ below. A proof can be simply obtained by modifying the proof of Lemma 4.8 with the removal of those terms involving $\bar{s}_{i j}, \bar{s}_{k i}$ and $\tau_{i}$. We skip the details. 
Lemma 4.3. Let $W=W_{A_{n-1}}$ and $f \in \mathbb{C}\left[x_{1}, \ldots, x_{n}\right]$. Then the following identity holds in $\ddot{\mathfrak{H}}_{W}^{\mathfrak{c}}$ :

$$
\left[y_{i}, f\right]=-u \sum_{k \neq i}\left(\frac{f-f^{s_{k i}}}{x_{i}-x_{k}}+\frac{f c_{k} c_{i}-c_{k} c_{i} f^{s_{k i}}}{x_{i}+x_{k}}\right) s_{k i} .
$$

Proof. It suffices to check the formula for every monomial $f$ of the form $x_{1}^{l_{1}} \cdots x_{n}^{l_{n}}$, which follows by Lemma 4.2 and an induction on $a$ based on the identity

$$
\left[y_{i}, x_{1}^{l_{1}} \cdots x_{a}^{l_{a}} x_{a+1}^{l_{a+1}}\right]=\left[y_{i}, x_{1}^{l_{1}} \cdots x_{a}^{l_{a}}\right] x_{a+1}^{l_{a+1}}+x_{1}^{l_{1}} \cdots x_{a}^{l_{a}}\left[y_{i}, x_{a+1}^{l_{a+1}}\right] .
$$

Now we are ready to compute the Dunkl operator for the $y_{i}$.

Theorem 4.4. Let $W=W_{A_{n-1}}$, and let $M$ be a $K$-module. The action of $y_{i}$ on the $\ddot{\mathfrak{H}}_{W}^{\mathfrak{c}}$-module $\mathbb{C}\left[x_{1}, \ldots, x_{n}\right] \otimes M$ is realized as a Dunkl operator as follows. For any polynomial $f \in \mathbb{C}\left[x_{1}, \ldots, x_{n}\right]$ and $m \in M$, we have

$$
y_{i} \circ(f \otimes m)=-u \sum_{k \neq i}\left(\frac{f-f^{s_{k i}}}{x_{i}-x_{k}}+\frac{f c_{k} c_{i}-c_{k} c_{i} f^{s_{k i}}}{x_{i}+x_{k}}\right) \otimes s_{k i} m .
$$

Proof. We calculate that $y_{i} \circ(f \otimes m)=\left[y_{i}, f\right] \otimes m+f \otimes y_{i} m=\left[y_{i}, f\right] \otimes m$. Now the result follows from Lemma 4.3.

Lemma 4.5. Let $W=W_{A_{n-1}}$. Then the following holds in $\ddot{\mathfrak{H}}_{W}^{\mathfrak{c}}$ for $l \in \mathbb{Z}_{+}$and $i \neq j$ :

$$
\left[y_{j}^{l}, x_{i}\right]=u \frac{y_{j}^{l}-y_{i}^{l}}{y_{j}-y_{i}}\left(1+c_{j} c_{i}\right) s_{i j} \quad \text { and } \quad\left[y_{i}^{l}, x_{i}\right]=-u \sum_{k \neq i} \frac{y_{i}^{l}-y_{k}^{l}}{y_{i}-y_{k}}\left(1+c_{k} c_{i}\right) s_{k i} \text {. }
$$

Proof. This lemma is a type $A$ counterpart of Lemma 4.11 for type $B$ below, with the removal of those terms involving $\bar{s}_{i j}, \bar{s}_{k i}$ and $\tau_{i}$. We omit the details.

Lemma 4.6. Let $W=W_{A_{n-1}}$, and let $f \in \mathbb{C}\left[y_{1}, \ldots, y_{n}\right]$. Then the following identity holds in $\ddot{\mathfrak{H}}_{W}^{\mathfrak{c}}$ :

$$
\left[f, x_{i}\right]=-u \sum_{k \neq i} \frac{f-f^{s_{k i}}}{y_{i}-y_{k}}\left(1+c_{k} c_{i}\right) s_{k i} .
$$

Proof. It suffices to check the formula for every monomial $f$, which can be done as for the formula in Lemma 4.3, now using Lemma 4.5 instead of Lemma 4.2.

Now we are ready to compute the Dunkl operator for the $x_{i}$.

Theorem 4.7. Let $W=W_{A_{n-1}}$, and let $M$ be a $K$-module. The action of $x_{i}$ on $\mathbb{C}\left[y_{1}, \ldots, y_{n}\right] \otimes M$ is realized as a Dunkl operator as follows. For any polynomial $f \in \mathbb{C}\left[y_{1}, \ldots, y_{n}\right]$ and $m \in M$, we have

$$
x_{i} \circ(f \otimes m)=u \sum_{k \neq i} \frac{f-f^{s_{k i}}}{y_{i}-y_{k}} \otimes\left(1+c_{k} c_{i}\right) s_{k i} m .
$$


Proof. We observe that

$$
x_{i} \circ(f \otimes m)=\left[x_{i}, f\right] \otimes m+f \otimes x_{i} m=\left[x_{i}, f\right] \otimes m .
$$

Now the result follows by Lemma 4.6.

The Dunkl operators for $\ddot{\mathfrak{H}}_{\boldsymbol{B}_{n}}^{\mathfrak{c}}$. We first prepare a few lemmas. The proofs of Lemmas 4.8, 4.9, and 4.11 appear in the appendix.

Lemma 4.8. Let $W=W_{B_{n}}$. Then the following holds in $\ddot{\mathfrak{H}}_{W}^{\mathfrak{c}}$ for $l \in \mathbb{Z}_{+}$and $i \neq j$ :

$$
\begin{gathered}
{\left[y_{i}, x_{j}^{l}\right]=u\left(\frac{x_{j}^{l}-x_{i}^{l}}{x_{j}-x_{i}}+\frac{x_{j}^{l}-\left(-x_{i}\right)^{l}}{x_{j}+x_{i}} c_{i} c_{j}\right) s_{i j}-u\left(\frac{x_{j}^{l}-\left(-x_{i}\right)^{l}}{x_{j}+x_{i}}-\frac{x_{j}^{l}-x_{i}^{l}}{x_{j}-x_{i}} c_{i} c_{j}\right) \bar{s}_{i j},} \\
\begin{aligned}
{\left[y_{i}, x_{i}^{l}\right]=-u \sum_{k \neq i}\left(\frac{x_{i}^{l}-x_{k}^{l}}{x_{i}-x_{k}}+\frac{x_{i}^{l}-\left(-x_{k}\right)^{l}}{x_{i}+x_{k}} c_{k} c_{i}\right) s_{k i} } \\
\quad-u \sum_{k \neq i}\left(\frac{x_{i}^{l}-\left(-x_{k}\right)^{l}}{x_{i}+x_{k}}-\frac{x_{i}^{l}-x_{k}^{l}}{x_{i}-x_{k}} c_{k} c_{i}\right) \bar{s}_{k i}-\sqrt{2} v \frac{x_{i}^{l}-\left(-x_{i}\right)^{l}}{2 x_{i}} \tau_{i} .
\end{aligned}
\end{gathered}
$$

Lemma 4.9. Let $W=W_{B_{n}}$, and let $f \in \mathbb{C}\left[x_{1}, \ldots, x_{n}\right]$. Then the following holds in $\ddot{\mathfrak{H}}_{W}^{\mathfrak{c}}$ :

$$
\begin{aligned}
{\left[y_{i}, f\right]=-u \sum_{k \neq i}\left(\frac{f-f^{s_{k i}}}{x_{i}-x_{k}}\right.} & \left.+\frac{f-f^{\bar{s}_{k i}}}{x_{i}+x_{k}} c_{k} c_{i}\right) s_{k i} \\
& -u \sum_{k \neq i}\left(\frac{f-f^{\bar{s}_{k i}}}{x_{i}+x_{k}}-\frac{f-f^{s_{k i}}}{x_{i}-x_{k}} c_{k} c_{i}\right) \bar{s}_{k i}-\sqrt{2} v \frac{f-f^{\tau_{i}}}{2 x_{i}} \tau_{i} .
\end{aligned}
$$

Now we are ready to compute the Dunkl operator for the $y_{i}$.

Theorem 4.10. Let $W=W_{B_{n}}$, and let $M$ be a $K$-module. The action of $y_{i}$ on $\mathbb{C}\left[x_{1}, \ldots, x_{n}\right] \otimes M$ is realized as follows. For any polynomial $f \in \mathbb{C}\left[x_{1}, \ldots, x_{n}\right]$ and $m \in M$, we have

$$
\begin{aligned}
y_{i} \circ(f \otimes m)=- & u \sum_{k \neq i}\left(\frac{f-f^{s_{k i}}}{x_{i}-x_{k}}+\frac{f-f^{\bar{s}_{k i}}}{x_{i}+x_{k}} c_{k} c_{i}\right) \otimes s_{k i} m \\
& -u \sum_{k \neq i}\left(\frac{f-f^{\bar{s}_{k i}}}{x_{i}+x_{k}}-\frac{f-f^{s_{k i}}}{x_{i}-x_{k}} c_{k} c_{i}\right) \otimes \bar{s}_{k i} m-\sqrt{2} v \frac{f-f^{\tau_{i}}}{2 x_{i}} \otimes \tau_{i} m .
\end{aligned}
$$

Proof. We observe that

$$
y_{i} \circ(f \otimes m)=\left[y_{i}, f\right] \otimes m+f \otimes y_{i} m=\left[y_{i}, f\right] \otimes m .
$$

Now the result follows from Lemma 4.9. 
Lemma 4.11. Let $W=W_{B_{n}}$. Then the following hold in $\ddot{\mathfrak{H}}_{W}^{\mathfrak{c}}$ for $l \in \mathbb{Z}_{+}$and $i \neq j$ :

$$
\begin{aligned}
{\left[y_{j}^{l}, x_{i}\right] } & =u\left(\frac{y_{j}^{l}-y_{i}^{l}}{y_{j}-y_{i}}\left(1+c_{j} c_{i}\right) s_{i j}-\frac{y_{j}^{l}-\left(-y_{i}\right)^{l}}{y_{j}+y_{i}}\left(1-c_{j} c_{i}\right) \bar{s}_{i j}\right) . \\
{\left[y_{i}^{l}, x_{i}\right] } & =-u \sum_{k \neq i} \frac{y_{i}^{l}-y_{k}^{l}}{y_{i}-y_{k}}\left(1+c_{k} c_{i}\right) s_{k i}-u \sum_{k \neq i} \frac{y_{i}^{l}-\left(-y_{k}\right)^{l}}{y_{i}+y_{k}}\left(1-c_{k} c_{i}\right) \bar{s}_{k i} \\
-\sqrt{2} v \frac{y_{i}^{l}-\left(-y_{i}\right)^{l}}{2 y_{i}} & \tau_{i} .
\end{aligned}
$$

In a similar fashion, we can derive the next lemma from Lemma 4.11.

Lemma 4.12. Let $W=W_{B_{n}}$, and let $f \in \mathbb{C}\left[y_{1}, \ldots, y_{n}\right]$. Then the following identity holds in $\ddot{\mathfrak{H}}_{W}^{\mathfrak{c}}$ :

$$
\begin{aligned}
& {\left[f, x_{i}\right]=-u \sum_{k \neq i} \frac{f-f^{s_{k i}}}{y_{i}-y_{k}}\left(1+c_{k} c_{i}\right) s_{k i}-u \sum_{k \neq i} \frac{f-f^{s_{k i}}}{y_{i}+y_{k}}\left(1-c_{k} c_{i}\right) \bar{s}_{k i} } \\
&-\sqrt{2} v \frac{f-f^{\tau_{i}}}{2 y_{i}} \tau_{i} .
\end{aligned}
$$

Now we are ready to compute the Dunkl operator for the $x_{i}$.

Theorem 4.13. Let $W=W_{B_{n}}$. The action of $x_{i}$ on $\mathbb{C}\left[y_{1}, \ldots, y_{n}\right] \otimes M$ is realized as follows. For any polynomial $f \in \mathbb{C}\left[y_{1}, \ldots, y_{n}\right]$ and $m \in M$, we have

$$
\begin{array}{r}
x_{i} \circ(f \otimes m)=u \sum_{k \neq i} \frac{f-f^{s_{k i}}}{y_{i}-y_{k}} \otimes\left(1+c_{k} c_{i}\right) s_{k i} m+u \sum_{k \neq i} \frac{f-f^{\bar{s}_{k i}}}{y_{i}+y_{k}} \otimes\left(1-c_{k} c_{i}\right) \bar{s}_{k i} m \\
+\sqrt{2} v \frac{f-f^{\tau_{i}}}{2 y_{i}} \otimes \tau_{i} m .
\end{array}
$$

Proof. We observe that $x_{i} \circ(f \otimes m)=\left[x_{i}, f\right] \otimes m+f \otimes x_{i} m=\left[x_{i}, f\right] \otimes m$. Now the result follows from Lemma 4.12.

The Dunkl operators for $\ddot{\mathfrak{H}}_{D_{n}}^{\mathfrak{c}}$. Below, the actions of the $x_{i}$ and the $y_{i}$ are realized as Dunkl operators. Due to the similarity of the bracket relations $[\cdot, \cdot]$ in $\ddot{\mathfrak{H}}_{D_{n}}^{\mathfrak{c}}$ and $\ddot{\mathfrak{H}}_{B_{n}}^{\mathfrak{c}}$ (for instance, compare the type $D$ relation (3-3b) with the type $B$ relation (3-4b)), the formulas below for type $D_{n}$ are obtained from their type $B_{n}$ counterparts in the previous subsection by dropping the terms involving the parameter $v$. The proofs are the same as for the type $B$, and thus will be skipped.

Lemma 4.14. Let $W=W_{D_{n}}$, and $f \in \mathbb{C}\left[x_{1}, \ldots, x_{n}\right]$. Then the following holds in $\ddot{\mathfrak{H}}_{W}^{\mathfrak{c}}$ :

$$
\left[y_{i}, f\right]=-u \sum_{k \neq i}\left(\frac{f-f^{s_{k i}}}{x_{i}-x_{k}}+\frac{f-f^{\bar{s}_{k i}}}{x_{i}+x_{k}} c_{k} c_{i}\right) s_{k i}-u \sum_{k \neq i}\left(\frac{f-f^{\bar{s}_{k i}}}{x_{i}+x_{k}}-\frac{f-f^{s_{k i}}}{x_{i}-x_{k}} c_{k} c_{i}\right) \bar{s}_{k i} .
$$


Lemma 4.15. Let $W=W_{D_{n}}$, and $f \in \mathbb{C}\left[y_{1}, \ldots, y_{n}\right]$. Then the following identity holds in $\ddot{\mathfrak{H}}_{W}^{\mathfrak{c}}$ :

$$
\left[f, x_{i}\right]=-u \sum_{k \neq i} \frac{f-f^{s_{k i}}}{y_{i}-y_{k}}\left(1+c_{k} c_{i}\right) s_{k i}-u \sum_{k \neq i} \frac{f-f^{\bar{s}_{k i}}}{y_{i}+y_{k}}\left(1-c_{k} c_{i}\right) \bar{s}_{k i} .
$$

Theorem 4.16. Let $W=W_{D_{n}}$, and let $M$ be a $K$-module. The action of $y_{i}$ on $\mathbb{C}\left[x_{1}, \ldots, x_{n}\right] \otimes M$ is realized as a Dunkl operator as follows. For any polynomial $f \in \mathbb{C}\left[x_{1}, \ldots, x_{n}\right]$ and $m \in M$, we have

$$
\begin{aligned}
y_{i} \circ(f \otimes m)=-u \sum_{k \neq i}\left(\frac{f-f^{s_{k i}}}{x_{i}-x_{k}}+\frac{f-f^{\bar{s}_{k i}}}{x_{i}+x_{k}} c_{k} c_{i}\right) \otimes s_{k i} m \\
\quad-u \sum_{k \neq i}\left(\frac{f-f^{\bar{s}_{k i}}}{x_{i}+x_{k}}-\frac{f-f^{s_{k i}}}{x_{i}-x_{k}} c_{k} c_{i}\right) \otimes \bar{s}_{k i} m .
\end{aligned}
$$

Theorem 4.17. Let $W=W_{D_{n}}$, and let $M$ be a $K$-module. The action of $x_{i}$ on $\mathbb{C}\left[y_{1}, \ldots, y_{n}\right] \otimes M$ is realized as follows. For any $f \in \mathbb{C}\left[y_{1}, \ldots, y_{n}\right]$ and $m \in M$,

$$
x_{i} \circ(f \otimes m)=u \sum_{k \neq i} \frac{f-f^{s_{k i}}}{y_{i}-y_{k}} \otimes\left(1+c_{k} c_{i}\right) s_{k i} m+u \sum_{k \neq i} \frac{f-f^{\bar{s}_{k i}}}{y_{i}+y_{k}} \otimes\left(1-c_{k} c_{i}\right) \bar{s}_{k i} m .
$$

The even center for $\ddot{\mathfrak{H}}_{W}^{\mathfrak{c}}$. Recall that the even center $\mathscr{L}(A)$ of a superalgebra $A$ consists of the even central elements of $A$. It turns out that the algebra $\ddot{\mathfrak{H}}_{W}^{\mathfrak{c}}$ has a large center.

Proposition 4.18. Let $W$ be $W_{A_{n-1}}, W_{D_{n}}$ or $W_{B_{n}}$. The even center $\mathscr{\mathscr { H }}\left(\ddot{\mathfrak{H}}_{W}^{\mathfrak{c}}\right)$ contains $\mathbb{C}\left[y_{1}, \ldots, y_{n}\right]^{W}$ and $\mathbb{C}\left[x_{1}^{2}, \ldots, x_{n}^{2}\right]^{W}$ as subalgebras. In particular, $\ddot{\mathfrak{H}}_{W}^{\mathfrak{c}}$ is modulefinite over its even center.

Proof. Let $f \in \mathbb{C}\left[y_{1}, \ldots, y_{n}\right]^{W}$. Then by the definition of $\ddot{\mathfrak{H}}_{W}^{\mathfrak{c}}, f$ commutes with $\mathscr{C}_{n}, W$, and $y_{i}$ for all $1 \leq i \leq n$. Since $f=f^{w}$ for all $w \in W$, it follows by Lemmas 4.6, 4.12 or 4.15 (for type $A, D$ or $B$, respectively) that $\left[f, x_{i}\right]=0$ for each $i$. Hence $f$ commutes with $\mathscr{C}_{n}, W$, and $\mathbb{C}\left[x_{1}, \ldots, x_{n}\right]$. Therefore $f$ is in the even center $\mathscr{L}\left(\ddot{\mathfrak{H}}_{W}^{\mathfrak{c}}\right)$.

Suppose now that $f \in \mathbb{C}\left[x_{1}^{2}, \ldots, x_{n}^{2}\right]^{W}$. Then by the definition of $\ddot{\mathfrak{H}}_{W}^{\mathfrak{c}}, f$ commutes with $\mathscr{C}_{n}, W$, and $x_{i}$ for all $1 \leq i \leq n$. By Lemma 4.3, 4.9 or 4.14 (for type $A, D$ or $B$ respectively), we have $\left[y_{i}, f\right]=0$ for each $i$. Therefore $f$ is in the even center.

The module-finiteness over the even center now follows from the PBW property of $\ddot{\mathfrak{H}}_{W}^{\mathfrak{c}}$ (see Theorem 3.5). 


\section{Rational spin double affine Hecke algebras (sDaHa)}

In this section, we introduce the rational spin double affine Hecke algebras associated to the Weyl group $W$ of type $A_{n-1}, D_{n}$ and $B_{n}$, and then establish their PBW property.

Elements in $\mathbb{C} W^{-}$of order 2. Recall that the spin group algebra $\mathbb{C} W^{-}$has a presentation with generator $t_{i}$ given in Section 2. Introduce the notations

$$
t_{i \uparrow j}=\left\{\begin{array}{ll}
t_{i} t_{i+1} \cdots t_{j} & \text { if } i \leq j, \\
1 & \text { otherwise, }
\end{array} \quad t_{i \downarrow j}= \begin{cases}t_{i} t_{i-1} \cdots t_{j} & \text { if } i \geq j, \\
1 & \text { otherwise. }\end{cases}\right.
$$

Define the following odd elements in $\mathbb{C} W^{-}$of order 2 , which are analogs of reflections in $W$, for $1 \leq i<j \leq n$ :

$$
\begin{aligned}
t_{i j} & \equiv[i, j]=(-1)^{j-i-1} t_{j-1} \ldots t_{i+1} t_{i} t_{i+1} \ldots t_{j-1} \\
t_{j i} & \equiv[j, i]=-[i, j] \\
\bar{t}_{i j} & \equiv \overline{[i, j]}= \begin{cases}(-1)^{j-i-1} t_{j \uparrow n-1} t_{i \uparrow n-2} t_{n} t_{n-2 \downarrow i} t_{n-1 \downarrow j} & \text { for type } D_{n}, \\
(-1)^{j-i} t_{j \uparrow n-1} t_{i \uparrow n-2} t_{n} t_{n-1} t_{n} t_{n-2 \downarrow i} t_{n-1 \downarrow j} & \text { for type } B_{n}\end{cases} \\
\bar{t}_{j i} & \equiv \overline{[j, i]}=\overline{[i, j]} \\
\bar{t}_{i} & \equiv \overline{[i]}=(-1)^{n-i} t_{i} \cdots t_{n-1} t_{n} t_{n-1} \cdots t_{i} \quad \text { for } 1 \leq i \leq n .
\end{aligned}
$$

Note the natural inclusions of algebras $\mathbb{C} W_{A_{n-1}}^{-} \leq \mathbb{C} W_{D_{n}}^{-} \leq \mathbb{C} W_{B_{n}}^{-}$. In particular, $t_{1}, \ldots, t_{n-1}$ and $t_{n} t_{n-1} t_{n}$ generate a subalgebra of $\mathbb{C} W_{B_{n}}^{-}$that is isomorphic to $\mathbb{C} W_{D_{n}}^{-}$(where $-t_{n} t_{n-1} t_{n}$ corresponds to the $n$-th generator for $\mathbb{C} W_{D_{n}}^{-}$). Hence, the notations $[i, j]$ and $\overline{[i, j]}$ here are consistent with such a subalgebra structure. Although we will not use it in this paper, we can show for $i<j$ that $\overline{[i, j]}=[j, n][i, n-1] t_{n}[i, n-1][j, n]$.

The algebra $\ddot{\mathfrak{H}}_{W}^{-}$of type $\boldsymbol{A}_{n-1}$. The following algebra, $\ddot{\mathfrak{H}}_{A_{n-1}}$, was introduced in [Wang 2006]. We recall the definition here for convenience in the subsequent subsections.

Definition 5.1. Let $u \in \mathbb{C}$, and let $W=W_{A_{n-1}}$. The rational spin double affine Hecke algebra of type $A_{n-1}$, denoted by $\ddot{\mathfrak{H}}_{W}^{-}$or $\ddot{\mathfrak{H}}_{A_{n-1}}^{-}$, is the algebra generated by $\xi_{i}$ and $y_{i}$ for $1 \leq i \leq n$ and $\mathbb{C} W^{-}$, subject to the relations

$$
\begin{aligned}
& \begin{cases}y_{i} y_{j}=y_{j} y_{i}, & \xi_{i} \xi_{j}=-\xi_{j} \xi_{i} \\
t_{i} y_{i}=y_{i+1} t_{i}, & t_{i} \xi_{i}=-\xi_{i+1} t_{i} \\
t_{i} y_{j}=y_{j} t_{i}, & t_{i} \xi_{j}=-\xi_{j} t_{i}\end{cases} \\
& {\left[y_{j}, \xi_{i}\right]=-u[i, j] \quad \text { for } i \neq j,} \\
& {\left[y_{i}, \xi_{i}\right]=u \sum_{k \neq i}[i, k] .}
\end{aligned}
$$




\section{The algebra $\ddot{\mathfrak{H}}_{W}^{-}$of type $D_{n}$.}

Definition 5.2. Let $u \in \mathbb{C}$, and let $W=W_{D_{n}}$. The rational spin double affine Hecke algebra of type $D_{n}$, denoted by $\ddot{\mathfrak{H}}_{W}^{-}$or $\ddot{\mathfrak{H}}_{D_{n}}^{-}$, is the algebra generated by $\xi_{i}, y_{i}$ for $1 \leq i \leq n$ and $\mathbb{C} W^{-}$, subject to (5-1) and the additional relations

$$
\begin{array}{rlrl}
t_{n} y_{n} & =-y_{n-1} t_{n}, & t_{n} \xi_{n} & =-\xi_{n-1} t_{n}, \\
t_{n} y_{j} & =y_{j} t_{n}, & & \\
t_{n} \xi_{j} & =-\xi_{j} t_{n}, \quad \text { for } j \neq n-1, n, \\
{\left[y_{j}, \xi_{i}\right]} & =-u[i, j]+u \overline{[i, j]} \quad \text { for } i \neq j, \\
{\left[y_{i}, \xi_{i}\right]} & =u \sum_{k \neq i}([i, k]+\overline{[i, k]}) .
\end{array}
$$

\section{The algebra $\ddot{\mathfrak{H}}_{W}^{-}$of type $B_{n}$.}

Definition 5.3. Let $u, v \in \mathbb{C}$, and let $W=W_{B_{n}}$. The rational spin double affine Hecke algebra of type $B_{n}$, denoted by $\ddot{\mathfrak{H}}_{W}^{-}$or $\ddot{\mathfrak{H}}_{B_{n}}^{-}$, is the algebra generated by $\xi_{i}$ and $y_{i}$ for $1 \leq i \leq n$ and $\mathbb{C} W_{B_{n}}^{-}$, subject to (5-1) and the additional relations

$$
\begin{array}{rlrl}
t_{n} y_{n} & =-y_{n} t_{n}, \quad t_{n} \xi_{n}=-\xi_{n} t_{n} & \\
t_{n} y_{j} & =y_{j} t_{n}, \quad t_{n} \xi_{j}=-\xi_{j} t_{n} & \text { for } j \neq n, \\
{\left[y_{j}, \xi_{i}\right]} & =-u[i, j]+u \overline{[i, j]} \quad \text { for } i \neq j, \\
{\left[y_{i}, \xi_{i}\right]} & =u \sum_{k \neq i}([i, k]+\overline{[i, k]})+v \overline{[i]} .
\end{array}
$$

If necessary, we write $\ddot{\mathfrak{H}}_{W}^{-}(u, v)$ for $\ddot{\mathfrak{H}}_{W}^{-}$to indicate the dependence on $u$ and $v$.

Isomorphism of superalgebras. The algebra $\ddot{\mathfrak{H}}_{W}^{-}$contains several distinguished subalgebras: the skew-polynomial algebra $\mathscr{C}\left[\xi_{1}, \ldots, \xi_{n}\right]$, the spin Weyl group algebra $\mathbb{C} W^{-}$, and the polynomial algebra $\mathbb{C}\left[y_{1}, \ldots, y_{n}\right]$. The algebra $\ddot{\mathfrak{H}}_{W}^{-}$has a superalgebra structure with $y_{i}$ even and $\xi_{i}$ and $t_{i}$ odd for all $i$.

Lemma 5.4. Let $W$ be one of the Weyl groups $W_{A_{n-1}}, W_{D_{n}}$ or $W_{B_{n}}$. The map $\Phi: \mathscr{C}_{n} \rtimes \mathbb{C} W \rightarrow \mathscr{C}_{n} \otimes \mathbb{C} W^{-}$(which, by Theorem 2.1, is an isomorphism) sends

$$
\begin{aligned}
\left(c_{k}-c_{i}\right) s_{i k} & \mapsto-\sqrt{-2}[k, i], \\
\left(c_{k}+c_{i}\right) \bar{s}_{i k} & \mapsto-\sqrt{-2}[\overline{[k, i]}, \\
c_{i} \tau_{i} & \mapsto-\sqrt{-1} \overline{[i]}
\end{aligned}
$$

for $i \neq k$, whenever it is applicable.

Proof. We may assume that $i>k$ without loss of generality.

We prove (5-2) by induction on $i$. First, (5-2) holds for $i=k+1$ by Theorem 2.1. Assuming that (5-2) holds for $i$, that is, $\Phi\left(\left(c_{k}-c_{i}\right) s_{i k}\right)=-\sqrt{-2}[k, i]$, we have 
by Theorem 2.1 and the definition of $[k, i]$ that

$$
\begin{aligned}
\Phi\left(\left(c_{k}-c_{i+1}\right) s_{i+1, k}\right) & =\Phi\left(s_{i}\left(c_{k}-c_{i}\right) s_{i k} s_{i}\right) \\
& =\left(-\sqrt{-1} \beta_{i} t_{i}\right)(-\sqrt{-2}[k, i])\left(-\sqrt{-1} \beta_{i} t_{i}\right) \\
& =\sqrt{-2} t_{i}[k, i] t_{i}=-\sqrt{-2}[k, i+1] .
\end{aligned}
$$

We now prove (5-4) by a similar downward induction on $i$, whose initial case $i=n$ is taken care of by Theorem 2.1. Assume that (5-4) holds for $i+1 \leq n$, that is, $\Phi\left(c_{i+1} \tau_{i+1}\right)=-\sqrt{-1} \overline{[i+1]}$. Then, by Theorem 2.1 and the definition of $\overline{[i]}$,

$$
\begin{aligned}
\Phi\left(c_{i} \tau_{i}\right) & =\Phi\left(s_{i} c_{i+1} \tau_{i+1} s_{i}\right) \\
& =\left(-\sqrt{-1} \beta_{i} t_{i}\right)(-\sqrt{-1} \overline{[i+1]})\left(-\sqrt{-1} \beta_{i} t_{i}\right) \\
& =\sqrt{-1} t_{i} \overline{[i+1]} t_{i}=-\sqrt{-1} \overline{[i]} .
\end{aligned}
$$

Next, we prove (5-3) by downward induction, first on $k$ and then on $i$, for $W=W_{D_{n}}$. The initial case $i=n$ and $k=n-1$ holds by Theorem 2.1. Then, it follows by the induction assumption that $\Phi\left(\left(c_{k+1}+c_{n}\right) \bar{s}_{n, k+1}\right)=-\sqrt{-2} \overline{[k+1, n]}$; it follows by Theorem 2.1 and the definition of $\overline{[k, n]}$ that

$$
\begin{aligned}
\Phi\left(\left(c_{k}+c_{n}\right) \bar{s}_{n k}\right) & =\Phi\left(s_{k}\left(c_{k+1}+c_{n}\right) \bar{s}_{n, k+1} s_{k}\right) \\
& =\left(-\sqrt{-1} \beta_{k} t_{k}\right) \cdot(-\sqrt{-2} \overline{[k+1, n]}) \cdot\left(-\sqrt{-1} \beta_{k} t_{k}\right) \\
& =\sqrt{-2} t_{k} \overline{[k+1, n]} t_{k}=-\sqrt{-2} \overline{[k, n]} .
\end{aligned}
$$

This in turn becomes the initial step when $i=n$ for proving (5-3) by downward induction on $i$ (with fixed $k<n$ ). By the induction assumption, (5-3) holds for $i>k+1$. Then

$$
\begin{aligned}
\Phi\left(\left(c_{k}+c_{i-1}\right) \bar{s}_{i-1, k}\right) & =\Phi\left(s_{i-1}\left(c_{k}+c_{i}\right) \bar{s}_{i k} s_{i-1}\right) \\
& =\left(-\sqrt{-1} \beta_{i-1} t_{i-1}\right)(-\sqrt{-2} \overline{[k, i]})\left(-\sqrt{-1} \beta_{i-1} t_{i-1}\right) \\
& =\sqrt{-2} t_{i-1} \overline{[k, i]} t_{i-1}=-\sqrt{-2} \overline{[k, i-1]} .
\end{aligned}
$$

This completes the proof of (5-3) for type $D$.

The formula (5-3) for $W=W_{B_{n}}$ is similarly proved by double downward inductions on $k$ and then on $i$. The only difference from the type $D$ case is that for type $B$ we have to check the initial case when $k=n-1$ and $i=n$, which uses (5-2) and (5-4):

$$
\begin{aligned}
\Phi\left(\left(c_{n-1}+c_{n}\right) \bar{s}_{n-1, n}\right) & =\Phi\left(\tau_{n}\left(c_{k+1}-c_{n}\right) s_{n-1, n} \tau_{n}\right) \\
& =\left(-\sqrt{-1} c_{n} t_{n}\right) \cdot\left(-\sqrt{-2} t_{n-1}\right) \cdot\left(-\sqrt{-1} c_{n} t_{n}\right) \\
& =\sqrt{-2} t_{n} t_{n-1} t_{n}=-\sqrt{-2} \overline{[n-1, n]} .
\end{aligned}
$$

Thus the lemma is proved. 
Recall the isomorphism $\Phi: \mathscr{C}_{n} \rtimes \mathbb{C} W \rightarrow \mathscr{C}_{n} \otimes \mathbb{C} W^{-}$of superalgebras and its inverse $\Psi$ given in Theorem 2.1 .

Theorem 5.5. Let $W$ be one of the Weyl groups $W_{A_{n-1}}, W_{D_{n}}$ or $W_{B_{n}}$.

(1) There exists an isomorphism

$$
\Phi: \ddot{\mathfrak{H}}_{W}^{\mathfrak{c}} \longrightarrow \mathscr{C}_{n} \otimes \ddot{\mathfrak{H}}_{W}^{-}
$$

of superalgebras that extends $\Phi: \mathscr{C}_{n} \rtimes \mathbb{C} W \rightarrow \mathscr{C}_{n} \otimes \mathbb{C} W^{-}$and sends

$$
y_{i} \mapsto y_{i}, \quad x_{i} \mapsto \sqrt{-2} c_{i} \xi_{i}, \quad s_{i} \mapsto-\sqrt{-1} \beta_{i} t_{i}, \quad c_{i} \mapsto c_{i} \quad \text { for all } i .
$$

(2) The inverse

$$
\Psi: \mathscr{C}_{n} \otimes \ddot{\mathfrak{H}}_{W}^{-} \longrightarrow \ddot{\mathfrak{H}}_{W}^{\mathfrak{c}}
$$

extends $\Psi: \mathscr{C}_{n} \otimes \mathbb{C} W^{-} \rightarrow \mathscr{C}_{n} \rtimes \mathbb{C} W$ and sends

$$
y_{i} \mapsto y_{i}, \quad \xi_{i} \mapsto \frac{c_{i} x_{i}}{\sqrt{-2}}, \quad t_{i} \mapsto \sqrt{-1} \beta_{i} s_{i}, \quad c_{i} \mapsto c_{i} \quad \text { for all } i .
$$

In the terminology of [Wang 2007], $\ddot{\mathfrak{H}}_{W}^{\mathfrak{c}}$ and $\ddot{\mathfrak{H}}_{W}^{-}$are Morita superequivalent by Theorem 5.5.

Proof. Recall that $\Phi$ extends the isomorphism $\mathscr{C}_{n} \rtimes \mathbb{C W} \stackrel{\simeq}{\longrightarrow} \mathscr{C}_{n} \otimes \mathbb{C} W^{-}$. Among all the relations (3-1)-(3-4) for $\ddot{\mathfrak{H}}_{W}^{\mathfrak{c}}$, it is easy to check that those in (3-1) are preserved by $\Phi$. So it remains to check that $\Phi$ preserves the relations in (3-2), (3-3), (3-4) for $W=W_{A_{n-1}}, W_{D_{n}}$, and $W_{B_{n}}$, respectively.

We shall verify in detail that $\Phi$ preserves (3-4) with $W=W_{B_{n}}$. Indeed, by Lemma 5.4, we have for $i \neq j$ that

$$
\begin{aligned}
\Phi(\text { left side of }(3-4 a)) & =\sqrt{-2}\left[y_{j}, c_{i} \xi_{i}\right] \\
& =\sqrt{-2} c_{i}(-u[i, j]+u \overline{[i, j]}) \\
& =\Phi\left(u\left(\left(1+c_{j} c_{i}\right) s_{j i}-\left(1-c_{j} c_{i}\right) \bar{s}_{i j}\right)\right) \\
& =\Phi(\text { right side of }(3-4 a)) .
\end{aligned}
$$

Also, by Lemma 5.4, we have

$$
\begin{aligned}
\Phi(\text { left side of }(3-4 b)) & =\sqrt{-2}\left[y_{i}, c_{i} \xi_{i}\right] \\
& =\sqrt{-2} u c_{i} \sum_{k \neq i}([i, k]+\overline{[i, k]})+\sqrt{-2} v c_{i} \overline{[i]} \\
& =\Phi\left(-u \sum_{k \neq i}\left(\left(1+c_{k} c_{i}\right) s_{k i}+\left(1-c_{k} c_{i}\right) \bar{s}_{k i}\right)-\sqrt{2} v \tau_{i}\right) \\
& =\Phi(\text { right side of }(3-4 b)) .
\end{aligned}
$$

By dropping the terms involving $v$ in the above equations, we verify that the relations (3-3) with $W=W_{D_{n}}$ are preserved by $\Phi$. By further dropping the terms involving $\overline{[i j]}, \bar{s}_{i j}$ and so on, we can also verify (3-2) with $W=W_{A_{n-1}}$. 
So, the homomorphism $\Phi$ is well defined. Similarly, one shows that $\Psi$ is a well-defined algebra homomorphism. For example, the relation $t_{n} \xi_{n}=-\xi_{n-1} t_{n}$ in $\ddot{\mathfrak{H}}_{W}^{-}$for $W=W_{D_{n}}$ is preserved by $\Psi$, since

$$
\begin{aligned}
\Psi\left(t_{n} \xi_{n}\right) & =\frac{\sqrt{-1}}{\sqrt{2}}\left(c_{n-1}+c_{n}\right) s_{n} \frac{1}{\sqrt{-2}} c_{n} x_{n} \\
& =\frac{1}{2}\left(c_{n-1}+c_{n}\right) c_{n-1} x_{n-1} s_{n} \\
& =\frac{1}{2} c_{n-1} x_{n-1}\left(-c_{n-1}-c_{n}\right) s_{n}=-\Psi\left(\xi_{n-1} t_{n}\right) .
\end{aligned}
$$

On the other hand, the relation $t_{n} \xi_{n}=-\xi_{n} t_{n}$ in $\ddot{\mathfrak{H}}_{W}^{-}$for $W=W_{B_{n}}$ is preserved by $\Psi$, since

$$
\Psi\left(t_{n} \xi_{n}\right)=\sqrt{-1} c_{n} s_{n} \frac{1}{\sqrt{-2}} c_{n} x_{n}=\frac{1}{\sqrt{2}} x_{n} s_{n}=-\frac{1}{\sqrt{2}} c_{n} x_{n} c_{n} s_{n}=-\Psi\left(\xi_{n} t_{n}\right) .
$$

Since $\Phi$ and $\Psi$ are inverses on generators, they are (inverse) algebra isomorphisms.

The PBW property for $\ddot{\mathfrak{H}}_{W}^{-}$. We have the following PBW type property for the algebra $\ddot{\mathfrak{H}}_{W}^{-}$.

Theorem 5.6. Let $W$ be one of the Weyl groups $W_{A_{n-1}}, W_{D_{n}}$ or $W_{B_{n}}$. The multiplication of the subalgebras induces an isomorphism of vector spaces

$$
\mathscr{C}\left[\xi_{1}, \ldots, \xi_{n}\right] \otimes \mathbb{C} W^{-} \otimes \mathbb{C}\left[y_{1}, \ldots, y_{n}\right] \rightarrow \ddot{\mathfrak{H}}_{W}^{-} .
$$

Equivalently, the set $\left\{\xi^{\alpha} \sigma y^{\gamma}\right\}$ forms a basis for $\ddot{\mathfrak{H}}_{W}^{-}$, where $\sigma$ runs over a basis for $\mathbb{C} W^{-}$, and $\alpha, \gamma \in \mathbb{Z}_{+}^{n}$.

Proof. It follows from the defining relations for $\ddot{\mathfrak{H}}_{W}^{-}$that $\ddot{\mathfrak{H}}_{W}^{-}$is spanned by the elements $\xi^{\alpha} \sigma y^{\gamma}$, where $\sigma$ runs over a basis for $\mathbb{C} W^{-}$, and $\alpha, \gamma \in \mathbb{Z}_{+}^{n}$. By the isomorphism $\Psi: \mathscr{C}_{n} \otimes \ddot{\mathfrak{H}}_{W}^{-} \rightarrow \ddot{\mathfrak{H}}_{W}^{c}$ in Theorem 5.5, we see that the images $\Psi\left(\xi^{\alpha} \sigma y^{\gamma}\right)$ are linearly independent in $\ddot{\mathfrak{H}}_{W}^{\mathfrak{c}}$ by the PBW property for $\ddot{\mathfrak{H}}_{W}^{\mathfrak{c}}$ (see Theorem 3.5). So the elements $\xi^{\alpha} \sigma y^{\gamma}$ are linearly independent in $\ddot{\mathfrak{H}}_{W}^{-}$.

Therefore, the set $\left\{\xi^{\alpha} \sigma y^{\gamma}\right\}$ forms a basis for $\ddot{\mathfrak{H}}_{W}^{-}$.

The tensor product in the above theorem gives a triangular decomposition of the algebra $\ddot{\mathfrak{H}}_{W}^{-}$.

\section{The Dunkl operators for sDaHa}

Denote by $\mathfrak{h}_{\xi}$ the subalgebra of $\ddot{\mathfrak{H}}_{W}^{-}$generated by the $\xi_{i}$ for $1 \leq i \leq n$ and $\mathbb{C} W^{-}$. For a $\mathbb{C} W^{-}$-module $V$, it can be extended to a $\mathfrak{h}_{\xi^{-}}$modules by letting the actions of the $\xi_{i}$ on $V$ be trivial. We define the induced $\ddot{\mathfrak{H}}_{W}^{-}$-module

$$
V_{\xi}:=\ddot{\mathfrak{H}}_{W}^{-} \otimes_{\mathfrak{h}_{\xi}} V \cong C\left[y_{1}, \ldots, y_{n}\right] \otimes V .
$$


We will always identify $V_{\xi}=\mathbb{C}\left[y_{1}, \ldots, y_{n}\right] \otimes V$. On $\mathbb{C}\left[y_{1}, \ldots, y_{n}\right] \otimes V$, the element $t_{i} \in \mathbb{C} W^{-}$acts as $s_{i} \otimes t_{i}$, the element $y_{i}$ acts by left multiplication, and $\xi_{i}$ acts as a Dunkl operator, which we will describe in this section.

Under Lemma 5.4 and the superalgebra isomorphism $\Phi: \ddot{\mathfrak{H}}_{W}^{\mathfrak{c}} \rightarrow \mathscr{C}_{n} \otimes \ddot{\mathfrak{H}}_{W}^{-}$in Theorem 5.5, the results in this section are fairly straightforward counterparts of those in Section 4, and we omit the proofs.

The Dunkl operator for $\ddot{\mathfrak{H}}_{\boldsymbol{A}_{n-1}}$. The next lemma is the counterpart of Lemma 4.6. Lemma 6.1. Let $W=W_{A_{n-1}}$, and $f \in \mathbb{C}\left[y_{1}, \ldots, y_{n}\right]$. Then the following identity holds in $\ddot{\mathfrak{H}}_{W}^{-}$:

$$
\left[f, \xi_{i}\right]=-u \sum_{k \neq i} \frac{f-f^{s_{k i}}}{y_{i}-y_{k}}[k, i] .
$$

The following is the counterpart of Theorem 4.7.

Proposition 6.2. Let $W=W_{A_{n-1}}$, and let $V$ be a $\mathbb{C} W^{-}$-module. The action of $\xi_{i}$ on $\mathbb{C}\left[y_{1}, \ldots, y_{n}\right] \otimes V$ is realized as a Dunkl operator as follows. For any polynomial $f \in \mathbb{C}\left[y_{1}, \ldots, y_{n}\right]$ and $v \in V$, we have

$$
\xi_{i} \circ(f \otimes v)=u \sum_{k \neq i} \frac{f-f^{s_{k i}}}{y_{i}-y_{k}} \otimes[k, i] v .
$$

The Dunkl operator for $\ddot{\mathfrak{H}}_{\boldsymbol{B}_{\boldsymbol{n}}}^{-}$. This lemma is the counterpart of Lemma 4.11:

Lemma 6.3. Let $W=W_{B_{n}}$ and $l \in \mathbb{Z}_{+}$. Then

$$
\begin{aligned}
{\left[y_{j}^{l}, \xi_{i}\right] } & =u \frac{y_{j}^{l}-y_{i}^{l}}{y_{j}-y_{i}}[j, i]+u \frac{y_{j}^{l}-\left(-y_{i}\right)^{l}}{y_{j}+y_{i}} \overline{[j, i]} . \\
{\left[y_{i}^{l}, \xi_{i}\right] } & =-u \sum_{k \neq i} \frac{y_{i}^{l}-y_{k}^{l}}{y_{i}-y_{k}}[k, i]+u \sum_{k \neq i} \frac{y_{i}^{l}-\left(-y_{k}\right)^{l}}{y_{i}+y_{k}} \overline{[k, i]}+v \frac{y_{i}^{l}-\left(-y_{i}\right)^{l}}{2 y_{i}} \overline{[i]} .
\end{aligned}
$$

This lemma is the counterpart of Lemma 4.12:

Lemma 6.4. Let $W=W_{B_{n}}$. The following identity holds in $\ddot{\mathfrak{H}}_{W}^{-}$:

$$
[f, \xi]=-u \sum_{k \neq i} \frac{f-f^{s_{k i}}}{y_{i}-y_{k}}[k, i]+u \sum_{k \neq i} \frac{f-f^{\bar{s}_{k i}}}{y_{i}+y_{k}} \overline{[k, i]}+v \frac{f-f^{\tau_{i}}}{2 y_{i}} \overline{[i]} .
$$

This proposition is the counterpart of Theorem 4.13:

Proposition 6.5. Let $W=W_{B_{n}}$, and let $V$ be a $\mathbb{C} W^{-}$-module. The action of $\xi_{i}$ on $\mathbb{C}\left[y_{1}, \ldots, y_{n}\right] \otimes V$ is realized as a Dunkl operator as follows. For any polynomial $f \in \mathbb{C}\left[y_{1}, \ldots, y_{n}\right]$ and $m \in V$, we have

$\xi_{i} \circ(f \otimes m)=u \sum_{k \neq i} \frac{f-f^{s_{k i}}}{y_{i}-y_{k}} \otimes[k, i] m-u \sum_{k \neq i} \frac{f-f^{\bar{s}_{k i}}}{y_{i}+y_{k}} \otimes \overline{[k, i]} m-v \frac{f-f^{\tau_{i}}}{2 y_{i}} \otimes \overline{[i]} m$. 
The Dunkl operator for $\ddot{\mathfrak{H}}_{D_{n}}^{-}$.

Proposition 6.6. Let $W=W_{D_{n}}$, and let $V$ be a $\mathbb{C} W^{-}$-module. The action of $\xi_{i}$ on $\mathbb{C}\left[y_{1}, \ldots, y_{n}\right] \otimes V$ is realized as a Dunkl operator as follows. For any polynomial $f \in \mathbb{C}\left[y_{1}, \ldots, y_{n}\right]$ and $v \in V$,

$$
\xi_{i} \circ(f \otimes v)=u \sum_{k \neq i} \frac{f-f^{s_{k i}}}{y_{i}-y_{k}} \otimes[k, i] v-u \sum_{k \neq i} \frac{f-f^{\bar{s}_{k i}}}{y_{i}+y_{k}} \otimes \overline{[k, i]} v .
$$

The even center for $\ddot{\mathfrak{H}}_{W}^{-}$.

Proposition 6.7. Let $W$ be one of the Weyl groups $W_{A_{n-1}}, W_{D_{n}}$ or $W_{B_{n}}$. The even center for $\ddot{\mathfrak{H}}_{W}^{-}$contains $\mathbb{C}\left[y_{1}, \ldots, y_{n}\right]^{W}$ and $\mathbb{C}\left[\xi_{1}^{2}, \ldots, \xi_{n}^{2}\right]^{W}$. In particular, $\ddot{\mathfrak{H}}_{W}^{-}$is module-finite over its even center.

Proof.

By the isomorphism $\Phi: \ddot{\mathfrak{H}}_{W}^{\mathfrak{c}} \rightarrow \mathscr{C}_{n} \otimes \ddot{\mathfrak{H}}_{W}^{-}$(see Theorem 5.5) and Proposition 4.18, we have

$$
\begin{aligned}
& \mathbb{C}\left[y_{1}, \ldots, y_{n}\right]^{W} \subseteq \Phi\left(Z\left(\ddot{\mathfrak{H}}_{W}^{\mathfrak{c}}\right)\right)=Z\left(\mathscr{C}_{n} \otimes \ddot{\mathfrak{H}}_{W}^{-}\right), \\
& \mathbb{C}\left[\xi_{1}^{2}, \ldots, \xi_{n}^{2}\right]^{W} \subseteq \Phi\left(Z\left(\ddot{\mathfrak{H}}_{W}^{\mathfrak{c}}\right)\right)=Z\left(\mathscr{C}_{n} \otimes \ddot{\mathfrak{H}}_{W}^{-}\right) .
\end{aligned}
$$

The first statement follows by noting that $\mathbb{C}\left[y_{1}, \ldots, y_{n}\right]^{W}$ and $\mathbb{C}\left[\xi_{1}^{2}, \ldots, \xi_{n}^{2}\right]^{W}$ actually lie in $\ddot{\mathfrak{H}}_{W}^{-}$. The second statement now follows from the PBW property of $\ddot{\mathfrak{H}}_{W}^{-}$ (see Theorem 5.6).

\section{Rational covering double affine Hecke algebras (cDaHa)}

This section introduces the rational covering double affine Hecke algebras (cDaHa) $\ddot{\mathfrak{H}}_{W}^{\sim}$ associated to classical Weyl groups $W$. It has as its natural quotients the usual rational DaHa $\ddot{\mathfrak{H}}_{W}$ [Etingof and Ginzburg 2002] (which will be recalled below) and the rational sDaHa $\ddot{\mathfrak{H}}_{W}^{-}$introduced in Section 5.

“Reflections" in $\widetilde{W}$. Recall from Section 2.1 the distinguished double cover $\widetilde{W}$ of a Weyl group $W$ with generators $\tilde{t}_{i}$.

Introduce the notations

$$
\tilde{t}_{i \uparrow j}=\left\{\begin{array}{ll}
\tilde{t}_{i} \tilde{t}_{i+1} \cdots \tilde{t}_{j} & \text { if } i \leq j, \\
1 & \text { otherwise, }
\end{array} \quad \tilde{t}_{i \downarrow j}= \begin{cases}\tilde{t}_{i} \tilde{t}_{i-1} \cdots \tilde{t}_{j} & \text { if } i \geq j \\
1 & \text { otherwise. }\end{cases}\right.
$$


Define the following elements in $\widetilde{W}$, which are distinguished preimages of reflections in $W$ under the canonical map $\widetilde{W} \rightarrow W$ for $1 \leq i<j \leq n$ :

$$
\begin{aligned}
\{i, j\} & =z^{j-i-1} \tilde{t}_{j-1} \ldots \tilde{t}_{i+1} \tilde{t}_{i} \tilde{t}_{i+1} \ldots \tilde{t}_{j-1}, \\
\{j, i\} & =z\{i, j\}, \\
\overline{\{i, j\}} & = \begin{cases}z^{j-i-1} \tilde{t}_{j \uparrow n-1} \tilde{t}_{i \uparrow n-2} \tilde{t}_{n} \tilde{t}_{n-2 \downarrow i} \tilde{t}_{n-1 \downarrow j} & \text { for type } D_{n}, \\
z^{j-i} \tilde{t}_{j \uparrow n-1} \tilde{t}_{i \uparrow n-2} \tilde{t}_{n} \tilde{t}_{n-1} \tilde{t}_{n} \tilde{t}_{n-2 \downarrow i} \tilde{t}_{n-1 \downarrow j} & \text { for type } B_{n},\end{cases} \\
\overline{\{j, i\}} & =\overline{\{i, j\}}, \\
\{i\} & =z^{n-i} \tilde{t}_{i} \cdots \tilde{t}_{n-1} \tilde{t}_{n} \tilde{t}_{n-1} \cdots \tilde{t}_{i} \quad \text { for } 1 \leq i \leq n .
\end{aligned}
$$

We have $\{i, j\} \in \widetilde{W}_{A_{n-1}}$ and $\overline{\{i, j\}} \in \widetilde{W}_{D_{n}}$ for $1 \leq i<j \leq n$, and $\{i\} \in \widetilde{W}_{B_{n}}$ for $1 \leq i \leq n$. We have a sequence of subgroups $\widetilde{W}_{A_{n-1}} \leq \widetilde{W}_{D_{n}} \leq \widetilde{W}_{B_{n}}$. The next lemma is straightforward from the definitions, and it helps to explain our choices of notations (recall $s_{i j}=(i, j)$ and $\bar{s}_{i j}=\overline{(i, j)}$ ).

Lemma 7.1. Let $W$ be $\widetilde{W}_{A_{n-1}}, \widetilde{W}_{D_{n}}$, or $\widetilde{W}_{B_{n}}$. For $i \neq j$, the canonical quotient map $\Upsilon_{+}: \mathbb{C} \widetilde{W} \rightarrow \mathbb{C} W$ sends

$$
\{i, j\} \mapsto(i, j), \quad \overline{\{i, j\}} \mapsto \overline{(i, j)}, \quad\{i\} \mapsto \tau_{i},
$$

and, again for $i \neq j$, the canonical quotient map $\Upsilon_{-}: \mathbb{C} \widetilde{W} \rightarrow \mathbb{C} W^{-}$sends

$$
\{i, j\} \mapsto[i, j], \quad \overline{\{i, j\}} \mapsto \overline{[i, j]}, \quad\{i\} \mapsto \overline{[i]}
$$

whenever it makes sense for the given $W$.

The rational Cherednik algebras. Recall that $\mathfrak{h}=\mathbb{C}^{n}$, and we have identified $\mathbb{C}[\mathfrak{h}]=\mathbb{C}\left[x_{1}, \ldots, x_{n}\right]$ and $\mathbb{C}\left[\mathfrak{h}^{*}\right]=\mathbb{C}\left[y_{1}, \ldots, y_{n}\right]$. Below we shall recall, in a more concrete form, the definition from [Etingof and Ginzburg 2002] of rational double affine Hecke algebras (also called rational Cherednik algebras) associated to the classical Weyl groups.

Let $t, u \in \mathbb{C}$. Let $W$ be one of the Weyl groups $W_{A_{n-1}}, W_{D_{n}}$, or $W_{B_{n}}$ respectively. The rational Cherednik algebra $\ddot{\mathfrak{H}}_{W}$ is the algebra generated by $x_{i}$ and $y_{i}$ for $1 \leq$ $i \leq n$ and $W$, subject to the common relations

$$
\begin{aligned}
& x_{i} x_{j}=x_{j} x_{i}, \quad y_{i} y_{j}=y_{j} y_{i} \quad \text { for all } i, j, \\
& \sigma x=x^{\sigma} \sigma, \quad \sigma y=y^{\sigma} \sigma \quad \text { for } \sigma \in W, x \in \mathfrak{h}^{*}, y \in \mathfrak{h}
\end{aligned}
$$

and the additional relations

$$
\left.\begin{array}{c}
{\left[y_{j}, x_{i}\right]=u s_{i j} \quad \text { for } i \neq j,} \\
{\left[y_{i}, x_{i}\right]=t \cdot 1-u \sum_{k \neq i} s_{k i},}
\end{array}\right\} \quad \text { for type } A,
$$




$$
\left.\begin{array}{l}
{\left[y_{j}, x_{i}\right]=u\left(s_{i j}-\bar{s}_{i j}\right) \quad \text { for } i \neq j,} \\
{\left[y_{i}, x_{i}\right]=t \cdot 1-u \sum_{k \neq i}\left(s_{k i}+\bar{s}_{k i}\right),}
\end{array}\right\} \quad \text { for type } D,
$$

The algebra $\ddot{\mathfrak{H}}_{W}$ has the following well-known PBW property: the multiplication of the subalgebras induces a vector space isomorphism

$$
\mathbb{C}\left[\mathfrak{h}^{*}\right] \otimes \mathbb{C} W \otimes \mathbb{C}[\mathfrak{h}] \stackrel{\simeq}{\longrightarrow} \ddot{\mathfrak{H}}_{W} .
$$

Equivalently, the set $\left\{x^{\alpha} w y^{\gamma} \mid \alpha, \gamma \in \mathbb{Z}_{+}^{n}, w \in W\right\}$ forms a PBW basis for $\ddot{\mathfrak{H}}_{W}$.

The rational covering double affine Hecke algebra $\ddot{\mathfrak{H}}_{W}^{\sim}$. Recall that the group $\widetilde{W}$ from Section 2 has the defining relations given in Table 1 , and $\widetilde{W}$ contains a central element $z$ of order 2 .

Definition 7.2. Let $W=W_{A_{n-1}}$, and let $t, u \in \mathbb{C}$. The rational covering double affine Hecke algebra of type $A_{n-1}$, denoted by $\ddot{\mathfrak{H}}_{W}^{\sim}$ or $\ddot{\mathfrak{H}}_{A_{n-1}}^{\sim}$, is the algebra generated by $\tilde{x}_{i}$ and $\tilde{y}_{i}$ for $1 \leq i \leq n$ and $z, \tilde{t}_{1}, \ldots, \tilde{t}_{n-1}$ subject to the relations for $\widetilde{W}$, and the following relations: $z$ is central and

$$
\begin{aligned}
& \left\{\begin{aligned}
\tilde{x}_{i} \tilde{x}_{j} & =z \tilde{x}_{j} \tilde{x}_{i}, \quad \tilde{y}_{i} \tilde{y}_{j}=\tilde{y}_{j} \tilde{y}_{i} \quad \text { for } i \neq j, \\
\tilde{t}_{i} \tilde{x}_{j} & =z \tilde{x}_{j} \tilde{t}_{i}, \quad \tilde{t}_{i} \tilde{y}_{j}=\tilde{y}_{j} \tilde{t}_{i} \quad \text { for } j \neq i, i+1, \\
\tilde{t}_{i} \tilde{x}_{i+1} & =z \tilde{x}_{i} \tilde{t}_{i}, \quad \tilde{t}_{i} \tilde{y}_{i+1}=\tilde{y}_{i} \tilde{t}_{i},
\end{aligned}\right. \\
& {\left[\tilde{y}_{j}, \tilde{x}_{i}\right]=u z\{i, j\} \quad \text { for } j \neq i,} \\
& {\left[\tilde{y}_{i}, \tilde{x}_{i}\right]=-u z \sum_{k \neq i}\{i, k\} .}
\end{aligned}
$$

Definition 7.3. Let $W=W_{D_{n}}$, and let $u \in \mathbb{C}$. The rational covering double affine Hecke algebra of type $D_{n}$, denoted by $\ddot{\mathfrak{H}}_{W}^{\sim}$ or $\ddot{\mathfrak{H}}_{D_{n}}$, is the algebra generated by $\tilde{x}_{i}$ and $\tilde{y}_{i}$ for $1 \leq i \leq n$ and $z, \tilde{t}_{1}, \ldots, \tilde{t}_{n}$, subject to the relations for $\widetilde{W}$, relations (7-1), and the additional relations that $z$ is central and

$$
\begin{aligned}
\tilde{t}_{n} \tilde{x}_{j} & =z \tilde{x}_{j} \tilde{t}_{n}, \quad \tilde{t}_{n} \tilde{y}_{j}=\tilde{y}_{j} \tilde{t}_{n} \quad \text { for } i \neq n-1, n, \\
\tilde{t}_{n} \tilde{x}_{n} & =-\tilde{x}_{n-1} \tilde{t}_{n}, \quad \tilde{t}_{n} \tilde{y}_{n}=-\tilde{y}_{n-1} \tilde{t}_{n}, \\
{\left[\tilde{y}_{j}, \tilde{x}_{i}\right] } & =u z(\{i, j\}-\overline{\{i, j\}}) \quad \text { for } j \neq i, \\
{\left[\tilde{y}_{i}, \tilde{x}_{i}\right] } & =-u z \sum_{k \neq i}(\{i, k\}+\overline{\{i, k\}}) .
\end{aligned}
$$

Definition 7.4. Let $W=W_{B_{n}}$, and let $u, v \in \mathbb{C}$. The rational covering double affine Hecke algebra of type $B_{n}$, denoted by $\ddot{\mathfrak{H}}_{W}^{\sim}$ or $\ddot{\mathfrak{H}}_{B_{n}}^{\sim}$, is the algebra generated by $\tilde{x}_{i}$ 
and $\tilde{y}_{i}$ for $1 \leq i \leq n$ and $z, \tilde{t}_{1}, \ldots, \tilde{t}_{n}$, subject to the relations for $\widetilde{W}$, relations (7-1), and the additional relations that $z$ is central and

$$
\begin{array}{rlrl}
\tilde{t}_{n} \tilde{x}_{i} & =z \tilde{x}_{i} \tilde{t}_{n}, & \tilde{t}_{n} \tilde{y}_{i}=\tilde{y}_{i} \tilde{t}_{n} \quad \text { for } i \neq n, \\
\tilde{t}_{n} \tilde{x}_{n} & =-\tilde{x}_{n} \tilde{t}_{n}, & \tilde{t}_{n} \tilde{y}_{n}=-\tilde{y}_{n} \tilde{t}_{n}, & \\
{\left[\tilde{y}_{j}, \tilde{x}_{i}\right]} & =u z(\{i, j\}-\overline{\{i, j\}}) & & \text { for } j \neq i, \\
{\left[\tilde{y}_{i}, \tilde{x}_{i}\right]} & =-u z \sum_{k \neq i}(\{i, k\}+\overline{\{i, k\}})-v z\{i\} . &
\end{array}
$$

PBW basis for $\ddot{\mathfrak{H}}_{W}$. The next result uses the notion of rational cDaHa to provide a link between the rational Cherednik algebra $\ddot{\mathfrak{H}}_{W}^{t=0}$ with the specialization $t=0$ and the rational sDaHa .

Proposition 7.5. Let $W=W_{A_{n-1}}, W_{D_{n}}$, or $W_{B_{n}}$. Then the quotient of the rational cDaHa $\ddot{\mathfrak{H}}_{W}^{\sim}$ by the ideal $\langle z-1\rangle$ (respectively, by the ideal $\langle z+1\rangle$ ) is isomorphic to the rational Cherednik algebra $\ddot{\mathfrak{H}}_{W}^{t=0}$ (respectively, the rational sDaHa $\ddot{\mathfrak{H}}_{W}^{-}$).

Proof. We will merely construct the isomorphisms of superalgebras explicitly, while noting that the verification follows directly from the definitions of the various algebras involved.

The canonical isomorphism map $\Upsilon_{+}: \mathbb{C} \widetilde{W} /\langle z-1\rangle \rightarrow \mathbb{C} W$ (see Lemma 7.1) can be extended to the isomorphism of superalgebras

$$
\Upsilon_{+}: \ddot{\mathfrak{H}}_{W}^{\sim} /\langle z-1\rangle \rightarrow \ddot{\mathfrak{H}}_{W}^{t=0}, \quad \tilde{t}_{i} \mapsto s_{i}, \quad \tilde{x}_{i} \mapsto x_{i}, \quad \tilde{y}_{i} \mapsto y_{i} .
$$

Also, the canonical isomorphism map $\Upsilon_{-}: \mathbb{C} \widetilde{W} /\langle z+1\rangle \rightarrow \mathbb{C} W^{-}$(see Lemma 7.1) can be extended to the isomorphism of superalgebras $\Upsilon_{+}: \ddot{\mathfrak{H}}_{W}^{\sim} /\langle z+1\rangle \rightarrow \ddot{\mathfrak{H}}_{W}^{-}$by sending $\tilde{t}_{i} \mapsto t_{i}, \quad \tilde{x}_{i} \mapsto \xi_{i}$ and $\tilde{y}_{i} \mapsto y_{i}$.

The next theorem follows from Proposition 7.5, the PBW basis theorem (Theorem 5.6) for $\ddot{\mathfrak{H}}_{W}^{-}$, and the PBW property for $\ddot{\mathfrak{H}}_{W}$ (see [Etingof and Ginzburg 2002]), by the same type of argument for [Wang 2007, Proposition 3.10] or [Khongsap and Wang 2008, Theorem 5.5].

Theorem 7.6. Let $W=W_{A_{n-1}}, W_{D_{n}}$, or $W_{B_{n}}$. Then the elements $\tilde{x}^{\alpha} \tilde{w} \tilde{y}^{\gamma}$, where $\alpha, \gamma \in \mathbb{Z}_{+}^{n}$ and $\tilde{w} \in \widetilde{W}$, form a basis for $\ddot{\mathfrak{H}}_{W}^{\sim}$.

\section{Appendix: Proofs of several lemmas}

Proof of Lemma 3.7. We will show that the relations (3-4) are invariant under the conjugation by elements $c_{l}$ for $1 \leq l \leq n$. The verifications for the invariants of other relations under the conjugation by $c_{l}$ are similar and will be omitted. 
Consider the relation (3-4a) first. Clearly, (3-4a) is invariant under the conjugation by $c_{l}$ for $l \neq i, j$. Moreover, we calculate that

$$
\begin{aligned}
c_{i}(\text { right side of }(3-4 \mathrm{a})) c_{i} & =u\left(\left(c_{i} c_{j}-1\right) s_{j i}-\left(-c_{i} c_{j}-1\right) \bar{s}_{i j}\right) \\
& =-\left[y_{j}, x_{i}\right]=c_{i}(\text { left side of }(3-4 \mathrm{a})) c_{i}, \\
c_{j}(\text { right side of }(3-4 \mathrm{a})) c_{j} & =u\left(\left(c_{j} c_{i}+1\right) s_{j i}-\left(-c_{j} c_{i}+1\right) \bar{s}_{i j}\right) \\
& =\left[y_{j}, x_{i}\right]=c_{j}(\text { left side of }(3-4 \mathrm{a})) c_{j} .
\end{aligned}
$$

Thus (3-4a) is invariant under conjugation by any $c_{l}$.

Next, we will show that the relation (3-4b) is invariant under the conjugation by each $c_{l}$. Indeed, we have

$c_{i}($ right side of $(3-4 b)) c_{i}$

$$
\begin{aligned}
& =-\sqrt{2} v c_{i} \tau_{i} c_{i}-u \sum_{k \neq i} c_{i}\left(\left(1+c_{k} c_{i}\right) s_{k i}+\left(1-c_{k} c_{i}\right) \bar{s}_{k i}\right) c_{i} \\
& =\sqrt{2} v \tau_{i}-u \sum_{k \neq i}\left(\left(c_{i} c_{k}-1\right) s_{k i}+\left(-c_{i} c_{k}-1\right) \bar{s}_{k i}\right) \\
& =\sqrt{2} v \tau_{i}+u \sum_{k \neq i}\left(\left(1+c_{k} c_{i}\right) s_{k i}+\left(1-c_{k} c_{i}\right) \bar{s}_{k i}\right) \\
& =-\left[y_{i}, x_{i}\right]=c_{i}(\text { left side of }(3-4 \mathrm{~b})) c_{i} .
\end{aligned}
$$

For $j \neq i$, we have

$$
\begin{gathered}
c_{j}(\text { right side of }(3-4 \mathrm{~b})) c_{j}=-\sqrt{2} v \tau_{i}-u c_{j}\left(\left(1+c_{j} c_{i}\right) s_{j i}+\left(1-c_{j} c_{i}\right) \bar{s}_{j i}\right) c_{j} \\
\quad-u \sum_{k \neq i, j} c_{j}\left(\left(1+c_{k} c_{i}\right) s_{k i}+\left(1-c_{k} c_{i}\right) \bar{s}_{k i}\right) c_{j} \\
=-\sqrt{2} v \tau_{i}-u\left(\left(c_{j} c_{i}+1\right) s_{j i}+\left(-c_{j} c_{i}+1\right) \bar{s}_{j i}\right) c_{j} \\
\quad-u \sum_{k \neq i, j}\left(\left(1+c_{k} c_{i}\right) s_{k i}+\left(1-c_{k} c_{i}\right) \bar{s}_{k i}\right) \\
\left.=c_{j} \text { (left side of }(3-4 \mathrm{~b})\right) c_{j} .
\end{gathered}
$$

Proof of Lemma 3.8. We will show below that the relations (3-4) are invariant under the conjugation by elements in $W_{B_{n}}$. The proof can be readily modified to yield the Weyl group invariance of the relations (3-2) and (3-3) in type $A$ and $D$ cases respectively, and we leave the details to the interested reader.

Case (i): We check the invariance of (3-4a) under $W_{B_{n}}$. Consider first the conjugation invariance by the transposition $s_{l k}$. If $\{l, k\} \cap\{i, j\}=\varnothing$, then

$s_{l k}($ right side of $(3-4 \mathrm{a})) s_{l k}=u\left(\left(1+c_{j} c_{i}\right) s_{j i}-\left(1-c_{j} c_{i}\right) \bar{s}_{i j}\right)$

$$
=\left[y_{j}, x_{i}\right]=s_{l k}(\text { left side of }(3-4 \mathrm{a})) s_{l k} .
$$

If $\{l, k\} \cap\{i, j\}=\{j\}$, then we may assume $l=j$ and we have

$s_{j k}($ right side of $(3-4 \mathrm{a})) s_{j k}=u\left(\left(1+c_{k} c_{i}\right) s_{i k}-\left(1-c_{k} c_{i}\right) \bar{s}_{i k}\right)$

$$
=\left[y_{k}, x_{i}\right]=s_{j k}(\text { left side of }(3-4 \mathrm{a})) s_{j k} .
$$


We leave an entirely analogous computation when $\{l, k\} \cap\{i, j\}=\{i\}$ to the reader.

Now, if $\{l, k\}=\{i, j\}$, then

$$
\begin{aligned}
s_{j i}(\text { right side of }(3-4 \mathrm{a})) s_{j i} & =u\left(\left(1+c_{i} c_{j}\right) s_{i j}-\left(1-c_{i} c_{j}\right) \bar{s}_{i j}\right) \\
& =\left[y_{i}, x_{j}\right]=s_{j i}(\text { left side of }(3-4 \mathrm{a})) s_{j i} .
\end{aligned}
$$

So (3-4a) is invariant under the conjugation by each transposition $s_{l k}$.

It remains to show that (3-4a) is invariant under the conjugation by the simple reflection $s_{n}=\tau_{n}$. Observe that (3-4a) is clearly invariant under conjugation by $s_{n}$ for $n \neq j, i$. Moreover, if $j=n$, then

$$
\begin{aligned}
s_{n}(\text { right side of }(3-4 \mathrm{a})) s_{n} & =u\left(\left(1-c_{j} c_{i}\right) \bar{s}_{j i}-\left(1+c_{j} c_{i}\right) s_{i j}\right) \\
& =-\left[y_{j}, x_{i}\right]=s_{n}(\text { left side of }(3-4 \mathrm{a})) s_{n} .
\end{aligned}
$$

If $i=n$, then

$$
\begin{aligned}
s_{n}(\text { right side of }(3-4 \mathrm{a})) s_{n} & =u\left(\left(1-c_{j} c_{i}\right) \bar{s}_{j i}-\left(1+c_{j} c_{i}\right) s_{i j}\right) \\
& =-\left[y_{j}, x_{i}\right]=s_{n}(\text { left side of }(3-4 \mathrm{a})) s_{n} .
\end{aligned}
$$

This completes Case (i).

Case (ii): We check the invariance of (3-4b) under $W_{B_{n}}$. Consider first the conjugation invariance by a transposition $s_{j l}$. If $\{j, l\} \cap\{i\}=\varnothing$, then

$$
\begin{aligned}
& s_{j l}(\text { right side of }(3-4 b)) s_{j l} \\
& \qquad \begin{array}{l}
=-u s_{j l}\left(\left(1+c_{j} c_{i}\right) s_{j i}+\left(1-c_{j} c_{i}\right) \bar{s}_{j i}\right) s_{j l} \\
\quad-u s_{j l}\left(\left(1+c_{l} c_{i}\right) s_{l i}+\left(1-c_{l} c_{i}\right) \bar{s}_{l i}\right) s_{j l} \\
\quad-u \sum_{k \neq i, j, l} s_{j l}\left(\left(1+c_{k} c_{i}\right) s_{k i}+\left(1-c_{k} c_{i}\right) \bar{s}_{k i}\right) s_{j l}-\sqrt{2} v \tau_{i} \\
=\left[y_{i}, x_{i}\right]=s_{j l}(\text { left side of }(3-4 \mathrm{~b})) s_{j l} .
\end{array}
\end{aligned}
$$

If $\{j, l\} \cap\{i\}=\{i\}$, we may assume that $j=i$, and then

$s_{i l}($ right side of $(3-4 b)) s_{i l}$

$$
\begin{aligned}
= & -u s_{i l}\left(\left(1+c_{l} c_{i}\right) s_{j i}+\left(1-c_{l} c_{i}\right) \bar{s}_{l i}\right) s_{i l} \\
& \quad-u \sum_{k \neq i, l} s_{i l}\left(\left(1+c_{k} c_{l}\right) s_{k l}+\left(1-c_{k} c_{l}\right) \bar{s}_{k l}\right) s_{i l}-\sqrt{2} v \tau_{l} \\
= & {\left[y_{l}, x_{l}\right]=s_{i l}(\text { left side of }(3-4 \mathrm{~b})) s_{i l} . }
\end{aligned}
$$


It remains to show that (3-4b) is invariant under the conjugation by the simple reflection $s_{n} \equiv \tau_{n} \in W_{B_{n}}$. If $i \neq n$, we have

$$
\begin{aligned}
& s_{n}(\text { right side of }(3-4 b)) s_{n} \\
& \begin{aligned}
=- & \sqrt{2} v \tau_{i}-u s_{n}\left(\left(1+c_{n} c_{i}\right) s_{n i}+\left(1-c_{n} c_{i}\right) \bar{s}_{n i}\right) s_{n} \\
& -u \sum_{k \neq i, n} s_{n}\left(\left(1+c_{k} c_{i}\right) s_{k i}+\left(1-c_{k} c_{i}\right) \bar{s}_{k i}\right) s_{n} \\
=- & \sqrt{2} v \tau_{i}-u\left(\left(1-c_{n} c_{i}\right) \bar{s}_{n i}+\left(1+c_{n} c_{i}\right) s_{n i}\right) \\
& -u \sum_{k \neq i, n}\left(\left(1+c_{k} c_{i}\right) s_{k i}+\left(1-c_{k} c_{i}\right) \bar{s}_{k i}\right) \\
= & {\left[y_{i}, x_{i}\right]=s_{n}(\text { left side of }(3-4 \mathrm{~b})) s_{n} . }
\end{aligned}
\end{aligned}
$$

If $i=n$, then

$$
\begin{aligned}
& s_{n}(\text { right side of }(3-4 b)) s_{n} \\
& \quad=-\sqrt{2} v \tau_{n}-u \sum_{k \neq n}\left(\left(1-c_{k} c_{n}\right) \bar{s}_{k n}+\left(1+c_{k} c_{n}\right) s_{k n}\right) \\
& \left.=\left[y_{n}, x_{n}\right]=s_{n} \text { (left side of }(3-4 \mathrm{~b})\right) s_{n} .
\end{aligned}
$$

This completes the proof of (ii). Hence the lemma is proved.

Proof of Lemma 3.9. We will establish the Jacobi identity for $W=W_{B_{n}}$. The proof can be easily modified for the cases of type $A$ and $D$, and we leave the details to the reader.

The Jacobi identity holds trivially among triple the $x_{i}$ or triple the $y_{i}$.

Now, we consider the triple with two $y$ 's and one $x$. The case with two identical $y_{i}$ is trivial. So we first consider $x_{i}, y_{j}$, and $y_{l}$ where $i, j, l$ are all distinct. The Jacobi identity holds in this case since

$$
\begin{aligned}
& {\left[x_{i},\left[y_{j}, y_{l}\right]\right]+\left[y_{l},\left[x_{i}, y_{j}\right]\right]+\left[y_{j},\left[y_{l}, x_{i}\right]\right]} \\
& =0+\left[y_{l},-u\left(\left(1+c_{j} c_{i}\right) s_{j i}-\left(1-c_{j} c_{i}\right) \bar{s}_{i j}\right)\right] \\
& +\left[y_{j}, u\left(\left(1+c_{l} c_{i}\right) s_{l i}-\left(1-c_{l} c_{i}\right) \bar{s}_{i l}\right)\right]=0 .
\end{aligned}
$$

Now for $i \neq j$, we have

$$
\begin{aligned}
{\left[x_{i},\left[y_{i}, y_{j}\right]\right]+} & {\left[y_{j},\left[x_{i}, y_{i}\right]\right]+\left[y_{i},\left[y_{j}, x_{i}\right]\right] } \\
=0+ & {\left[y_{j}, u \sum_{k \neq i}\left(\left(1+c_{k} c_{i}\right) s_{k i}+\left(1-c_{k} c_{i}\right) \bar{s}_{k i}\right)+\sqrt{2} v \tau_{i}\right] } \\
& +\left[y_{i}, u\left(\left(1+c_{j} c_{i}\right) s_{j i}-\left(1-c_{j} c_{i}\right) \bar{s}_{i j}\right)\right] \\
=[ & \left.y_{j}, u \sum_{k \neq i, j}\left(\left(1+c_{k} c_{i}\right) s_{k i}+\left(1-c_{k} c_{i}\right) \bar{s}_{k i}\right)\right] \\
& +\left[y_{j}, u\left(\left(1+c_{j} c_{i}\right) s_{j i}+\left(1-c_{j} c_{i}\right) \bar{s}_{j i}\right)\right] \\
& +\left[y_{i}, u\left(\left(1+c_{j} c_{i}\right) s_{j i}-\left(1-c_{j} c_{i}\right) \bar{s}_{i j}\right)\right] \\
=0 & +u\left(\left(1+c_{j} c_{i}\right) y_{j} s_{j i}+\left(1-c_{j} c_{i}\right) y_{j} \bar{s}_{j i}\right)
\end{aligned}
$$




$$
\begin{aligned}
& -u\left(\left(1+c_{j} c_{i}\right) s_{j i} y_{j}+\left(1-c_{j} c_{i}\right) \bar{s}_{j i} y_{j}\right) \\
& +u\left(\left(1+c_{j} c_{i}\right) y_{i} s_{j i}-\left(1-c_{j} c_{i}\right) y_{i} \bar{s}_{i j}\right) \\
& -u\left(\left(1+c_{j} c_{i}\right) s_{j i} y_{i}-\left(1-c_{j} c_{i}\right) \bar{s}_{i j} y_{i}\right)=0 .
\end{aligned}
$$

Now we consider the Jacobi identity with one $y$ and two $x$ 's. The case with all distinct indices can be easily verified as above. Moreover, for $i \neq j$, we have

$$
\begin{aligned}
{\left[x_{i},\right.} & {\left.\left[y_{i}, x_{j}\right]\right]+\left[x_{j},\left[x_{i}, y_{i}\right]\right]+\left[y_{i},\left[x_{j}, x_{i}\right]\right] } \\
= & {\left[x_{i}, u\left(\left(1+c_{i} c_{j}\right) s_{i j}-\left(1-c_{i} c_{j}\right) \bar{s}_{i j}\right)\right] } \\
& +\left[x_{j}, u \sum_{k \neq i}\left(\left(1+c_{k} c_{i}\right) s_{k i}+\left(1-c_{k} c_{i}\right) \bar{s}_{k i}\right)+\sqrt{2} v \tau_{i}\right]+0 \\
= & {\left[x_{i}, u\left(\left(1+c_{i} c_{j}\right) s_{i j}-\left(1-c_{i} c_{j}\right) \bar{s}_{i j}\right)\right] } \\
& +\left[x_{j}, u\left(\left(1+c_{j} c_{i}\right) s_{i j}+\left(1-c_{j} c_{i}\right) \bar{s}_{i j}\right)\right] \\
= & u\left(\left(1-c_{i} c_{j}\right) x_{i} s_{i j}-\left(1+c_{i} c_{j}\right) x_{i} \bar{s}_{i j}\right)-u\left(\left(1+c_{i} c_{j}\right) s_{i j} x_{i}-\left(1-c_{i} c_{j}\right) \bar{s}_{i j} x_{i}\right) \\
& +u\left(\left(1-c_{j} c_{i}\right) x_{j} s_{i j}+\left(1+c_{j} c_{i}\right) x_{j} \bar{s}_{i j}\right) \\
& -u\left(\left(1+c_{j} c_{i}\right) s_{i j} x_{j}+\left(1-c_{j} c_{i}\right) \bar{s}_{i j} x_{j}\right)=0 .
\end{aligned}
$$

This completes the verification of the Jacobi identity for any triples.

Proof of Lemma 4.8. We will proceed by induction. For $l=1$, then the equations hold by (3-4). Now assume that the statement is true for $l$. Then, for $i \neq j$

$$
\begin{aligned}
& {\left[y_{i}, x_{j}^{l+1}\right]=\left[y_{i}, x_{j}^{l}\right] x_{j}+x_{j}^{l}\left[y_{i}, x_{j}\right]} \\
& =u\left(\frac{x_{j}^{l}-x_{i}^{l}}{x_{j}-x_{i}}+\frac{x_{j}^{l}-\left(-x_{i}\right)^{l}}{x_{j}+x_{i}} c_{i} c_{j}\right) s_{i j} x_{j}-u\left(\frac{x_{j}^{l}-\left(-x_{i}\right)^{l}}{x_{j}+x_{i}}-\frac{x_{j}^{l}-x_{i}^{l}}{x_{j}-x_{i}} c_{i} c_{j}\right) \bar{s}_{i j} x_{j} \\
& +x_{j}^{l} u\left(\left(1+c_{i} c_{j}\right) s_{i j}-\left(1-c_{i} c_{j}\right) \bar{s}_{i j}\right) \\
& =u\left(\frac{x_{j}^{l+1}-x_{i}^{l+1}}{x_{j}-x_{i}}+\frac{x_{j}^{l+1}-\left(-x_{i}\right)^{l+1}}{x_{j}+x_{i}} c_{i} c_{j}\right) s_{i j} \\
& -u\left(\frac{x_{j}^{l+1}-\left(-x_{i}\right)^{l+1}}{x_{j}+x_{i}}-\frac{x_{j}^{l+1}-x_{i}^{l+1}}{x_{j}-x_{i}} c_{i} c_{j}\right) \bar{s}_{i j},
\end{aligned}
$$

and

$$
\begin{aligned}
{\left[y_{i}, x_{i}^{l+1}\right]=} & {\left[y_{i}, x_{i}^{l}\right] x_{i}+x_{i}^{l}\left[y_{i}, x_{i}\right] } \\
= & -u \sum_{k \neq i}\left(\frac{x_{i}^{l}-x_{k}^{l}}{x_{i}-x_{k}}+\frac{x_{i}^{l}-\left(-x_{k}\right)^{l}}{x_{i}+x_{k}} c_{k} c_{i}\right) s_{k i} x_{i} \\
& -u \sum_{k \neq i}\left(\frac{x_{i}^{l}-\left(-x_{k}\right)^{l}}{x_{i}+x_{k}}-\frac{x_{i}^{l}-x_{k}^{l}}{x_{i}-x_{k}} c_{k} c_{i}\right) \bar{s}_{k i} x_{i}-\sqrt{2} v \frac{x_{i}^{l} \tau_{i}-\tau_{i} x_{i}^{l}}{2 x_{i}} x_{i}
\end{aligned}
$$




$$
\begin{aligned}
& -u x_{i}^{l} \sum_{k \neq i}\left(\left(1+c_{k} c_{i}\right) s_{k i}+\left(1-c_{k} c_{i}\right) \bar{s}_{k i}\right)-\sqrt{2} v x_{i}^{l} \tau_{i} \\
= & -u \sum_{k \neq i}\left(\frac{x_{i}^{l+1}-x_{k}^{l+1}}{x_{i}-x_{k}}+\frac{x_{i}^{l+1}-\left(-x_{k}\right)^{l+1}}{x_{i}+x_{k}} c_{k} c_{i}\right) s_{k i} \\
& -u \sum_{k \neq i}\left(\frac{x_{i}^{l+1}-\left(-x_{k}\right)^{l+1}}{x_{i}+x_{k}}-\frac{x_{i}^{l+1}-x_{k}^{l+1}}{x_{i}-x_{k}} c_{k} c_{i}\right) \bar{s}_{k i} \\
-\sqrt{2} v & \frac{x_{i}^{l+1} \tau_{i}-\tau_{i} x_{i}^{l+1}}{2 x_{i}}
\end{aligned}
$$

Proof of Lemma 4.9. It suffices to check the formula for every monomial $f$. First, we consider the monomial $g=\prod_{j \neq i} x_{j}^{a_{j}}$. By induction and Lemma 4.8, we can show that the formula holds for the monomial of the form $g=\prod_{j \neq i} x_{j}^{a_{j}}$ (the detail of the induction step does not differ much from the following calculation). Now consider the monomial $f=x_{i}^{l} g$. By Lemma 4.8, we have

$$
\begin{aligned}
& {\left[y_{i}, f\right] }=\left[y_{i}, x_{i}^{l}\right] g+x_{i}^{l}\left[y_{i}, g\right] \\
&=-u \sum_{k \neq i}\left(\frac{x_{i}^{l}-x_{k}^{l}}{x_{i}-x_{k}}+\frac{x_{i}^{l}-\left(-x_{k}\right)^{l}}{x_{i}+x_{k}} c_{k} c_{i}\right) s_{k i} g \\
&-u \sum_{k \neq i}\left(\frac{x_{i}^{l}-\left(-x_{k}\right)^{l}}{x_{i}+x_{k}}-\frac{x_{i}^{l}-x_{k}^{l}}{x_{i}-x_{k}} c_{k} c_{i}\right) \bar{s}_{k i} g-\sqrt{2} v \frac{x_{i}^{l}-\left(-x_{i}\right)^{l}}{2 x_{i}} \tau_{i} g \\
&-u \sum_{k \neq i} x_{i}^{l}\left(\frac{g-g^{s_{k i}}}{x_{i}-x_{k}}+\frac{g-g^{\bar{s}_{k i}}}{x_{i}+x_{k}} c_{k} c_{i}\right) s_{k i}-u \sum_{k \neq i} x_{i}^{l}\left(\frac{g-g^{\bar{s}_{k i}}}{x_{i}+x_{k}}-\frac{g-g^{s_{k i}}}{x_{i}-x_{k}} c_{k} c_{i}\right) \bar{s}_{k i} \\
&=-u \sum_{k \neq i}\left(\frac{f-f^{s_{k i}}}{x_{i}-x_{k}}+\frac{f-f^{\bar{s}_{k i}}}{x_{i}+x_{k}} c_{k} c_{i}\right) s_{k i}-u \sum_{k \neq i}\left(\frac{f-f^{\bar{s}_{k i}}}{x_{i}+x_{k}}-\frac{f-f^{s_{k i}}}{x_{i}-x_{k}} c_{k} c_{i}\right) \bar{s}_{k i} \\
&-\sqrt{2} v \frac{f-f^{\tau_{i}}}{2 x_{i}} \tau_{i} .
\end{aligned}
$$

Proof of Lemma 4.11. We will proceed by induction. For $l=1$, the equations hold by (3-4). Now assume that the statement is true for $l$. Then, for $i \neq j$,

$$
\begin{aligned}
{\left[y_{j}^{l+1}, x_{i}\right]=} & y_{j}\left[y_{j}^{l}, x_{i}\right]+\left[y_{j}, x_{i}\right] y_{j}^{l} \\
=u y_{j}\left(\frac{y_{j}^{l}-y_{i}^{l}}{y_{j}-y_{i}}\left(1+c_{j} c_{i}\right) s_{i j}-\frac{y_{j}^{l}-\left(-y_{i}\right)^{l}}{y_{j}+y_{i}}\left(1-c_{j} c_{i}\right) \bar{s}_{i j}\right) & \quad+u\left(\left(1+c_{j} c_{i}\right) s_{j i}-\left(1-c_{j} c_{i}\right) \bar{s}_{i j}\right) y_{j}^{l} \\
& \\
= & u\left(\frac{y_{j}^{l+1}-y_{i}^{l+1}}{y_{j}-y_{i}}\left(1+c_{j} c_{i}\right) s_{i j}-\frac{y_{j}^{l+1}-\left(-y_{i}\right)^{l+1}}{y_{j}+y_{i}}\left(1-c_{j} c_{i}\right) \bar{s}_{i j}\right) .
\end{aligned}
$$


On the other hand, we have

$$
\begin{aligned}
& {\left[y_{i}^{l+1}, x_{i}\right]=y_{i}\left[y_{i}^{l}, x_{i}\right]+\left[y_{i}, x_{i}\right] y_{i}^{l}} \\
& =-u \sum_{k \neq i} y_{i} \frac{y_{i}^{l}-y_{k}^{l}}{y_{i}-y_{k}}\left(1+c_{k} c_{i}\right) s_{k i}-u \sum_{k \neq i} y_{i} \frac{y_{i}^{l}-\left(-y_{k}\right)^{l}}{y_{i}+y_{k}}\left(1-c_{k} c_{i}\right) \bar{s}_{k i} \\
& -\sqrt{2} v y_{i} \frac{y_{i}^{l}-\left(-y_{i}\right)^{l}}{2 y_{i}} \tau_{i} \\
& -u \sum_{k \neq i}\left(\left(1+c_{k} c_{i}\right) s_{k i}+\left(1-c_{k} c_{i}\right) \bar{s}_{k i}\right) y_{i}^{l}-\sqrt{2} v \tau_{i} y_{i}^{l} \\
& =-u \sum_{k \neq i} \frac{y_{i}^{l+1}-y_{k}^{l+1}}{y_{i}-y_{k}}\left(1+c_{k} c_{i}\right) s_{k i}-u \sum_{k \neq i} \frac{y_{i}^{l+1}-\left(-y_{k}\right)^{l+1}}{y_{i}+y_{k}}\left(1-c_{k} c_{i}\right) \bar{s}_{k i} \\
& -\sqrt{2} v \frac{y_{i}^{l+1}-\left(-y_{i}\right)^{l+1}}{2 y_{i}} \tau_{i}
\end{aligned}
$$

\section{References}

[Cherednik 2005] I. Cherednik, Double affine Hecke algebras, London Mathematical Society Lecture Note Series 319, Cambridge University Press, 2005. MR 2007e:32012 Zbl 1087.20003

[Chmutova 2005] T. Chmutova, "Twisted symplectic reflection algebras", preprint, 2005. arXiv math/0505653v1

[Drinfeld 1986] V. G. Drinfel'd, "Degenerate affine Hecke algebras and Yangians", Funktsional. Anal. i Prilozhen. 20:1 (1986), 69-70. In Russian; translated in Functional Anal. Appl. 20:1 (1986), 62-64. MR $87 \mathrm{~m}: 22044$

[Dunkl 1989] C. F. Dunkl, "Differential-difference operators associated to reflection groups", Trans. Amer. Math. Soc. 311:1 (1989), 167-183. MR 90k:33027 Zbl 0652.33004

[Etingof 2007] P. Etingof, "Lectures on Calogero-Moser systems", preprint, 2007. arXiv math/ $0606233 \mathrm{v} 3$

[Etingof and Ginzburg 2002] P. Etingof and V. Ginzburg, "Symplectic reflection algebras, CalogeroMoser space, and deformed Harish-Chandra homomorphism”, Invent. Math. 147:2 (2002), 243348. MR 2003b:16021 Zbl 1061.16032

[Gordon 2003] I. Gordon, "Baby Verma modules for rational Cherednik algebras", Bull. London Math. Soc. 35:3 (2003), 321-336. MR 2004c:16050 Zbl 1042.16017

[Karpilovsky 1987] G. Karpilovsky, The Schur multiplier, London Mathematical Society Monographs. New Series 2, Oxford University Press, 1987. MR 93j:20002 Zbl 0619.20001

[Khongsap and Wang 2008] T. Khongsap and W. Wang, "Hecke-Clifford algebras and spin Hecke algebras, I: The classical affine type", Transformation Groups 13:2 (2008), 389-412.

[Lusztig 1989] G. Lusztig, "Affine Hecke algebras and their graded version”, J. Amer. Math. Soc. 2:3 (1989), 599-635. MR 90e:16049 Zbl 0715.22020

[Lusztig 2002] G. Lusztig, "Cuspidal local systems and graded Hecke algebras, III", Represent. Theory 6 (2002), 202-242. MR 2004k:20010 Zbl 1031.22007

[Morris 1976] A. O. Morris, "Projective representations of reflection groups", Proc. London Math. Soc. (3) 32:3 (1976), 403-420. MR 58 \#5874 Zbl 0336.20007

[Nazarov 1997] M. Nazarov, "Young's symmetrizers for projective representations of the symmetric group”, Adv. Math. 127:2 (1997), 190-257. MR 98m:20019 Zbl 0930.20011 
[Rouquier 2005] R. Rouquier, "Representations of rational Cherednik algebras", pp. 103-131 in Infinite-dimensional aspects of representation theory and applications (Charlottesville, VA, 2004), edited by S. Berman et al., Contemp. Math. 392, Amer. Math. Society, Providence, RI, 2005. MR 2007d:20006

[Schur 1911] I. Schur, ""Uber die Darstellung der symmetrischen und der alternierenden Gruppe durch gebrochene lineare Substitutionen", J. für Math 139 (1911), 155-250. JFM 42.0154.02

[Wang 2006] W. Wang, "Double affine Hecke algebras for the spin symmetric group", preprint, 2006. arXiv math.RT/0608074v2

[Wang 2007] W. Wang, "Spin Hecke algebras of finite and affine types", Adv. Math. 212:2 (2007), 723-748. MR 2329318 Zbl 1139.20004

Received February 14, 2008. Revised August 6, 2008.

TA KHONGSAP

DEPARTMENT OF MATHEMATICS

P. O. BOX 400137

UNIVERSITY OF VIRGINIA

CHARLOTTESVILle, VA 22904-4137

UNITED STATES

tk7p@virginia.edu

http://people.virginia.edu/ tk7p

WEIQIANG WANG

DEPARTMENT OF MATHEMATICS

P. O. BOX 400137

UNIVERSITY OF VIRGINIA

CharlotTesville, VA 22904-4137

UNITED STATES

ww9c@virginia.edu

www.math.virginia.edu/ ww9c 